\title{
Production of multi-charmed hadrons by recombination in heavy ion collisions
}

\author{
Sungtae $\mathrm{Cho}^{1}$ and Su Houng Lee ${ }^{2}$ \\ ${ }^{1}$ Division of Science Education, Kangwon National University, Chuncheon 24341, Korea \\ ${ }^{2}$ Department of Physics and Institute of Physics and Applied Physics, Yonsei University, Seoul 03722, Korea
}

\begin{abstract}
We study the production of multi-charmed hadrons by recombination in heavy ion collisions by focusing on the production of $\Xi_{c c}, \Xi_{c c}^{*}, \Omega_{s c c}, \Omega_{s c c}^{*}, \Omega_{c c c}$ baryons and X(3872) mesons. Starting from the estimation of yields for those hadrons at chemical freeze-out in both the statistical and coalescence model, we evaluate their transverse momentum distributions at mid-rapidity in the coalescence model. We show that yields of multi-charmed hadrons in heavy ion collisions at RHIC and LHC are large enough, and thereby not only multi-charmed hadrons observed so far, e.g., the $\Xi_{c c}$ but also those which have not been observed yet, can be discovered sufficiently in heavy ion collisions. We also find that the transverse momentum distribution ratio between various multicharmed hadrons sensitively reflects the interplay between quark contents of corresponding hadrons as well as the transverse momentum distribution of charm quarks at the hadronization point, and therefore we insist that studying both the transverse momentum distributions of multi-charmed hadrons themselves and transverse momentum distribution ratios between various multi-charmed hadrons provide us with useful information on hadron production mechanism involving charm quarks in heavy ion collisions.
\end{abstract}

PACS numbers: 25.75.-q, 14.65.Dw, 13.60.Rj

\section{INTRODUCTION}

Relativistic heavy ion collision experiments have provided exclusive opportunities to study a system of quantum chromodynamic matter at high temperatures 1 [3]. Enormous energies available in heavy ion collisions allow the system to reach a phase transition temperature predicted by Lattice calculation [4], and as a result produce and sustain the so called quark-gluon plasma composed of deconfined quarks and gluons for a certain time during the experiments.

In order to investigate the properties of the quarkgluon plasma many probes have been proposed, and among others a heavy quark hadron has been considered to be one of the most useful probes in understanding not only the quark-gluon plasma properties but also the various aspects of phenomena in high energy heavy ion collisions. The possible suppression of the $J / \psi$ production caused by the color screening effects between charm and anti-charm quarks in the quark-gluon plasma has been suggested as one of the signatures for the formation of the system of free quarks and gluons in heavy ion collision experiments 5]. More recently, members of charmonium states such as $\psi(2 S)$ and $\chi_{c}$ mesons are known to play a role as an indicator of the temperature in the quark-gluon plasma phase since charmonium states with different binding energies are expected to dissociate at different temperatures in the system [6 $[8]$.

As the energies achievable in relativistic heavy ion collisions are increased, i.e., from $\sqrt{s_{N N}}=200 \mathrm{GeV}$ in $\mathrm{Au}-\mathrm{Au}$ collisions at the Relativistic Heavy Ion Collider (RHIC) to $\sqrt{s_{N N}}=2.76 \mathrm{TeV}$ in $\mathrm{Pb}-\mathrm{Pb}$ collisions at the Large Hadron Collider (LHC), or from $\sqrt{s_{N N}}=2.76$ $\mathrm{TeV}$ to $\sqrt{s_{N N}}=5.02 \mathrm{TeV}$ at LHC, heavy quarks are anticipated to be more abundantly produced than ever before, and consequently the possibilities of regenerating heavy quark hadrons from the quark-gluon plasma are also expected to be increased [9 11]. Therefore hadrons with heavy quarks become more important probes in investigating the properties of the quark-gluon plasma, so that it is necessary to understand in more detail the production of heavy quark hadrons in high energy heavy ion collisions. In that sense the coalescence model 12 16 which describes the production of hadrons as the process of coalescing constituent quarks in the quark-gluon plasma into hadrons using phase space density functions has become a more relevant tool in investigating the production of heavy quark hadrons.

In recent years the possibilities of observing various kinds of exotic hadrons with heavy quarks, e.g., the $X(3872), Z_{c}(3900), Z_{c}(4430)$, and $T_{c c}$ in heavy ion collision experiments have been considered based on the coalescence model [17 19]; it has been found that the yield of those exotic hadrons are large enough to be observed in heavy ion collisions, and the production yield of exotic hadrons is strongly dependent on their structures. However, we still find that production of even many normal hadrons with heavy quarks have not been taken into account yet in heavy ion collision experiments. Restricting our discussion to hadrons with charm quarks, we have not observed multi-charmed hadrons such as $\Xi_{c c}^{*}$, $\Omega_{s c c}, \Omega_{s c c}^{*}$, and $\Omega_{c c c}$ baryons even in elementary collisions though the existence of these charmed baryons was predicted long time ago based on the quark model with four flavors 20]. In regard to a doubly charmed baryon, the SELEX Collaboration reported the first observation of the $\Xi_{c c}$ baryon in elementary collisions in 2002 [21], which has not been confirmed yet, while very recently the LHCb Collaboration has reported the observation of the $\Xi_{c c}$ via the decay mode of $\Lambda_{c}^{+} K^{-} \pi^{+} \pi^{+}$also in elementary collisions [22].

By this reason, it is necessary to study the production 
of normal multi-charmed hadrons in relativistic heavy ion collisions, and therefore we discuss in this work the production of the $\Xi_{c c}, \Xi_{c c}^{*}, \Omega_{s c c}, \Omega_{s c c}^{*}$, and $\Omega_{c c c}$ baryon in heavy ion collisions. The production of the doubly charmed hadron, the $\Xi_{c c}$ has been considered previously in heavy ion collisions based on the Schoedinger equation [23], and also based on the Boltzmann transport equation [24]. Here, we focus on the production of multi-charmed hadrons including also the $\Xi_{c c}$ by recombination in heavy ion collisions, and consider both their yields and transverse momentum distributions.

We anticipate that when the mass of most doublycharmed hadrons are comparable to that of an exotic hadron, e.g., the $X(3872)$ meson, the yield of those hadron is also expected to be similar to or smaller than the yield of the $X(3872)$ meson. The $\Omega_{c c c}$ meson is an exception since it contains one more charm quark, and therefore the yield of the $\Omega_{c c c}$ is expected to be much smaller compared to that of the $X(3872)$ meson. Therefore, by comparing the yield of multi-charmed hadrons to that of exotic hadrons obtained in Ref. [19] we can estimate the possibility of measuring those multi-charmed hadrons in heavy ion collisions.

Here when comparing multi-charmed hadrons to exotic hadrons we restrict our discussion to the $X(3872)$ meson among many known exotic hadrons. The $X(3872)$, first discovered by Belle Collaboration in 2003 [25], is one of exotic hadrons whose structures have not been clearly understood. We still find various possibilities for the structure of the $X(3872)$ meson; a $\bar{D}^{0} D^{* 0}$ hadronic molecule, a pure charmonium state, a tetra-quark state, and a charmoniun-gluon hybrid state [26]. In order to understand the structure of the $X(3872)$ meson from the production in heavy ion collisions various investigations have already been performed including those involving the hadronic effects on the $X(3872)$ meson 27 29]. We also expect to understand better the structure of the $X(3872)$ meson in this work from studying the production of multi-charmed hadrons in heavy ion collisions.

We also consider the transverse momentum distribution of the $X(3872)$ meson as well as that of multicharmed hadrons. Since it has been found that the yield depends on the structure of hadrons, it is expected that transverse momentum distributions also depends on the structure of hadrons; we can obtain the yield after integrating the transverse momentum distribution over all transverse momenta. It has also been shown that transverse momentum distributions of charmonium states are dependent on their wave functions through the coalescence probability function, or the Wigner function [30]. Therefore by evaluating the transverse momentum distribution of multi-charmed hadrons we expect to obtain useful information on those hadrons, e.g., the dependence of the transverse momentum distribution on their constituent heavy quarks. Especially for the $X(3872)$ meson we consider two transverse momentum distributions of the $X(3872)$ assuming its structure to be either a four-quark state or a two-quark state. As two different structures will lead to two different transverse momentum distributions, future experimental measurements on transverse momentum distribution of the $X(3872)$ are expected to discriminate the structure of the $X(3872)$.

Moreover, based on transverse momentum distributions of multi-charmed hadrons we calculate the transverse momentum distribution ratio between various multi-charmed hadrons, and also the ratio between the $X(3872)$ and multi-charmed hadrons as has been done previously between the anti-proton and pion. The ratio between the anti-proton and pion is the baryon-tomeson ratio whereas the ratio between the $X(3872)$ meson and multi-charmed hadrons is the meson-to-baryon ratio. However, the ratio between the $X(3872)$ and $\Xi_{c c}$ will retain the same quark contents after cancelling the common quarks as in the ratio between the anti-proton and pion thereby enabling us to investigate the possibility of the enhanced production of the $X(3872)$ meson compared to the $\Xi_{c c}$ baryon.

Since the quark structure of the $X(3872)$ meson in a four-quark picture is considered to be $c \bar{c} q \bar{q}$, the $X(3872)$ meson is not a flavor exotic multi-charmed hadron, and therefore it is more appropriate to consider an explicit multi-charmed exotic hadron such as the $T_{c c}$ composed of $c c \bar{q} \bar{q}$ instead of the $X(3872)$. However, the $T_{c c}$ has not been observed experimentally yet, and moreover the yield and transverse momentum distribution of the $X(3872)$ is almost same as those of the $T_{c c}$ in the coalescence model under the condition that transverse momentum distributions of charm and anti-charm quarks are same. We only expect the slight difference between the transverse momentum distribution of the $X(3872)$ and that of the $T_{c c}$ at RHIC due to non-zero baryon chemical potential. Therefore, we discuss in detail the yield and transverse momentum distribution of the $T_{c c}$, but we focus more on the $X(3872)$ rather than the $T_{c c}$, and adopt the $X(3872)$ in comparing to normal multi-charmed hadrons.

The paper is organized as follows. In Sec. II, we first pay attention to the yields, and estimate the values of multi-charmed hadrons, the $\Xi_{c c}, \Xi_{c c}^{*}, \Omega_{s c c}, \Omega_{s c c}^{*}, \Omega_{c c c}$ baryon and the $\mathrm{X}(3872)$ meson at chemical freeze-out in both the statistical and coalescence model. We discuss also the various yield ratio between multi-charmed hadrons. In Sec. III, we focus on the transverse momentum distribution in heavy ion collisions, and evaluate that of multi-charmed hadrons mentioned above at mid-rapidity in the coalescence model. Then, we obtain the transverse momentum distribution ratios between multi-charmed hadrons, and investigate the dependence of those ratios on the quark contents, numbers of quarks, and so on in Sec. IV. Section V is devoted to conclusions. We show the equivalence of the transverse momentum distribution of a four-quark hadron on alternative relative coordinates in Appendix A. In the paper we use sometimes the simplified notation for the $X(3872)$ meson; $X_{4}$ for the $X(3872)$ meson in a four-quark state, and $X_{2}$ for the $X(3872)$ meson in a two-quark state. 


\section{PRODUCTION OF MULTI-CHARMED HADRONS FROM THE QUARK-GLUON PLASMA}

We evaluate yields of multi-charmed hadrons, $\Xi_{c c}, \Xi_{c c}^{*}$, $\Omega_{s c c}, \Omega_{s c c}^{*}, \Omega_{c c c}$ baryons as well as $X(3872)$ and $T_{c c}$ mesons produced in relativistic heavy ion collisions using both the statistical and coalescence model in midrapidity. The statistical hadronization model assuming hadron production in thermal and chemical equilibrium at chemical freeze-out, has been very successful in explaining the production yields of hadrons in heavy ion collisions [31]. In applying the statistical hadronization model here for the estimation of the production yields of multi-charmed hadrons we introduce additional charm quark fugacities, $\gamma_{c}$ in order to take into account charm quarks which are not in equilibrium in a quark-gluon plasma phase due to their heavier masses compared to available temperatures in a system. The yields are then given as

$$
N_{h}^{\text {stat }}=V_{H} \frac{g_{h}}{2 \pi^{2}} \int_{0}^{\infty} \frac{p^{2} d p}{\gamma_{c}^{-n} e^{E_{h} / T_{H}} \pm 1}
$$

where $g_{h}$ is the degeneracy factor of a hadron of species $h, n$ number of charm quarks in the hadron, $V_{H}$ and $T_{H}$ are the hadronization volume and temperature, respectively. $E_{h}=\sqrt{m_{h}^{2}+p^{2}}$ in Eq. (10) is the energy of the hadron of mass $m_{h}$. Here we consider the multi-charmed hadrons produced at the hadronization temperature and volume $T_{H}=162 \mathrm{MeV}$ and $V_{H}=2100 \mathrm{fm}^{3}$ at RHIC 32 and $T_{H}=156 \mathrm{MeV}$ and $V_{H}=5380 \mathrm{fm}^{3}$ at LHC [33], respectively. We assume that the total numbers of charm quarks available from the initial hard collisions are 4.1 at RHIC and 11 at LHC, which leads to the charm quark fugacity factors $\gamma_{c}=22$ at RHIC and 39 at LHC [19]. All charm quarks produced at the initial hard collisions are assumed to be conserved and fully distributed to charmed hadrons including $D, D^{*}, D_{s}$ mesons, and $\Lambda_{c}$ after chemical freeze-out [17-19].

We also consider yields of the $\Xi_{c c}, \Xi_{c c}^{*}, \Omega_{s c c}, \Omega_{s c c}^{*}, \Omega_{c c c}$, $X(3872)$ and $T_{c c}$ in the coalescence model which successfully explains the enhanced production of the baryon compared to the meson in the intermediate transverse momentum region [12 15]. Following Ref. 19] we assume that hadron productions by coalescence occur at the critical temperature $166 \mathrm{MeV}$ in the volume 1790 (3530) $\mathrm{fm}^{3}$ at RHIC (LHC) from quark constituents, light quarks, strange quarks, and charm quarks of their masses $350 \mathrm{MeV}, 500 \mathrm{MeV}$ and $1500 \mathrm{MeV}$, respectively. We also adopt that the number of light quarks available at hadronization is 302 (593), that of strange quarks 176 (347) and that of charm quarks 4.1 (11) at RHIC (LHC). Finally, by taking the charm quark oscillator frequencies for the Wigner function, $\omega_{c}=244 \mathrm{MeV}$ for RHIC and $278 \mathrm{MeV}$ for LHC we evaluate the production yields of the $\Xi_{c c}, \Omega_{s c c}, \Omega_{c c c}$ baryons, $T_{c c}$ and $X(3872)$ mesons, and show results in Table $\Pi$

We show two yields for the $X(3872)$ meson, one for the
TABLE I: The $\Xi_{c c}, \Xi_{c c}^{*}, \Omega_{s c c}, \Omega_{s c c}^{*}, \Omega_{c c c}, T_{c c}$ and $X(3872)$ yields at mid-rapidity in both the statistical and coalescence model expected at RHIC in $\sqrt{s_{N N}}=200 \mathrm{GeV} \mathrm{Au}+\mathrm{Au}$ collisions and at LHC in $\sqrt{s_{N N}}=2.76 \mathrm{TeV} \mathrm{Pb}+\mathrm{Pb}$ collisions.

\begin{tabular}{c|c|c|c|c}
\hline \hline & \multicolumn{2}{|c|}{ RHIC } & \multicolumn{2}{c}{ LHC } \\
\cline { 2 - 5 } & Stat. & Coal. & Stat. & Coal. \\
\hline$\Xi_{c c}$ & $1.0 \times 10^{-2}$ & $1.3 \times 10^{-3}$ & $2.8 \times 10^{-2}$ & $4.9 \times 10^{-3}$ \\
$\Xi_{c c}^{*}$ & $6.4 \times 10^{-3}$ & $9.0 \times 10^{-4}$ & $1.8 \times 10^{-2}$ & $3.3 \times 10^{-3}$ \\
$\Omega_{s c c}$ & $2.8 \times 10^{-3}$ & $2.5 \times 10^{-4}$ & $8.0 \times 10^{-3}$ & $9.0 \times 10^{-4}$ \\
$\Omega_{s c c}^{*}$ & $1.5 \times 10^{-3}$ & $1.6 \times 10^{-4}$ & $4.3 \times 10^{-3}$ & $6.0 \times 10^{-4}$ \\
$\Omega_{c c c}$ & $1.1 \times 10^{-4}$ & $1.1 \times 10^{-6}$ & $4.0 \times 10^{-4}$ & $5.3 \times 10^{-6}$ \\
$T_{c c}$ & $8.9 \times 10^{-4}$ & $5.3 \times 10^{-5}$ & $2.7 \times 10^{-3}$ & $1.3 \times 10^{-4}$ \\
$X_{2}$ & $5.7 \times 10^{-4}$ & $5.6 \times 10^{-4}$ & $1.7 \times 10^{-3}$ & $1.7 \times 10^{-3}$ \\
$X_{4}$ & $5.7 \times 10^{-4}$ & $5.3 \times 10^{-5}$ & $1.7 \times 10^{-3}$ & $1.3 \times 10^{-4}$ \\
\hline \hline
\end{tabular}

$X(3872)$ in a two-quark state, the $X_{2}$ and the other for the $X(3872)$ meson in a four-quark state, the $X_{4}$ [19]. We consider only a four-quark state for the $T_{c c}$ when evaluating the yield of the $T_{c c}$ in the coalescence model.

In the statistical hadronization model $3621.4 \mathrm{MeV}$ for the mass of the $\Xi_{c c}$ 22], $3648.0 \mathrm{MeV}$ for the $\Xi_{c c}^{*}, 3679.0$ $\mathrm{MeV}$ for the $\Omega_{s c c}, 3765.0 \mathrm{MeV}$ for the $\Omega_{s c c}^{*}, 4761.0 \mathrm{MeV}$ for the $\Omega_{c c c}$, 34], 3871.6 MeV for the $X(3872)$ [35], and $3797 \mathrm{MeV}$ for the $T_{c c}$ [19] are adopted. The mass of multi-charmed hadron taken here is very close to that obtained in the recent analysis [36]. When evaluating yields of the $\Xi_{c c}$ and $\Omega_{s c c}$, we have assumed the exclusive decay of a spin $3 / 2$ baryon to a spin $1 / 2$ baryon, similar to decay modes of $\Xi^{*}$ and $\Delta$ baryons [35] as summarized in the Table W. We expect that the $\Omega_{s c c}$ decays to the baryon with one charm quark like the $\Xi_{c}$ without decaying to the $\Xi_{c c}$. The $\Omega_{c c c}$ is expected to decay to either the $\Xi_{c c}$ or the $\Omega_{s c c}$, but the yield of the $\Omega_{c c c}$ is much smaller compared to those of the $\Xi_{c c}$ and $\Omega_{s c c}$, and therefore we neglect the contribution of the $\Omega_{c c c}$ decay to the yield of the $\Xi_{c c}$ and $\Omega_{s c c}$.

TABLE II: Assumed decay modes of $\Xi_{c c}^{*}$ and $\Omega_{s c c}^{*}$, baryons similar to the known decay modes of $\Delta$ and $\Xi^{*}$ baryons [35].

\begin{tabular}{c|c|c|c}
\hline \hline \multicolumn{2}{c|}{ Assumed decay modes } & \multicolumn{2}{|c}{ Similar decay modes } \\
\hline$\Xi_{c c}^{*} \rightarrow \Xi_{c c}$ & $100 \%$ & $\Delta \rightarrow N$ & $100 \%$ \\
$\Omega_{s c c}^{*} \rightarrow \Omega_{s c c}$ & $100 \%$ & $\Xi^{*} \rightarrow \Xi$ & $100 \%$ \\
\hline \hline
\end{tabular}

We see in Table \ that the yield decreases with increasing number of charm and light quarks in multicharmed hadrons in both the statistical and coalescence models, which is attributable to the smaller probability to combine much heavier and rarer charm quarks in the hadronization process; the yields of the triply charmed hadron, the $\Omega_{c c c}$ baryon and the four-quark hadron, the 
$X_{4}$ meson are smaller compared to those of the $\Xi_{c c}$ and $\Xi_{c c}^{*}$ baryon. On the other hand, we find that when the contribution from the $\Omega_{s c c}^{*}$ decay is not considered the yield of the $X_{4}$ is comparable to that of the $\Omega_{s c c}$ in the coalescence model, $8.2 \times 10^{-4}$ at RHIC and $3.0 \times 10^{-4}$ at LHC; forming two more light quarks of the constituent mass $350 \mathrm{MeV}$ is similar to constructing one more strange quark of the mass $500 \mathrm{MeV}$ in addition to two charm quarks. We also note that when the $X(3872)$ is considered to be a normal meson composed of a charm and and an anti-charm quark $X_{2}$, the yield in the coalescence model is almost same as that in the statistical model.

As shown in Table I, yields in the quark coalescence model are smaller than those in the statistical model, reflecting the suppression effects in the quark coalescence process [17-19]; the ratios of the yield in the coalescence model compared to that in the statistical model are 0.010 (0.013) for the $\Omega_{c c c}, 0.089(0.11)$ for the $\Omega_{s c c}, 0.13(0.18)$ for the $\Xi_{c c}, 0.060(0.048)$ for the $T_{c c}$, and $0.093(0.077)$ for the $X_{4}$ at RHIC (LHC).

We find in Table凹that the yield ratios between the $X_{4}$ and the $\Xi_{c c}$ are 0.056 (0.061) in the statistical model ,and 0.039 (0.027) in the coalescence model at RHIC (LHC), and that between the $X_{4}$ and the $\Omega_{s c c}$ are $0.20(0.21)$ in the statistical model and $0.21(0.15)$ in the coalescence model while that between $\Omega_{c c c}$ and the $\Xi_{c c}$ are 0.011 (0.014) in the statistical model and 0.00079 (0.0011) in the coalescence model at RHIC (LHC). We calculate the yield ratios between multi-charmed hadrons introduced in Table I, or the ratio between the yield of the heavier hadron and that of the lighter one, and summarized in Table III. We see that yield ratios involving the $\Omega_{c c c}$, or $\Omega_{c c c} / \Xi_{c c}, \Omega_{c c c} / \Omega_{s c c}$ and $\Omega_{c c c} / X_{4}$ at LHC are always larger than those at RHIC in both the statistical and coalescence model. Other ratios, except for the $\Omega_{c c c}$ ratios at RHIC, are comparable to or larger than those at LHC.

TABLE III: The yield ratios between multi-charmed hadrons, the $\Xi_{c c}, \Omega_{s c c}, \Omega_{c c c}$ baryon, and the $X(3872)$ meson at midrapidity in both the statistical and coalescence model expected at RHIC in $\sqrt{s_{N N}}=200 \mathrm{GeV} \mathrm{Au}+\mathrm{Au}$ collisions and at $\mathrm{LHC}$ in $\sqrt{s_{N N}}=2.76 \mathrm{TeV} \mathrm{Pb}+\mathrm{Pb}$ collisions.

\begin{tabular}{c|c|c|c|c|c|c}
\hline \hline \multirow{2}{*}{ Stat. } & \multicolumn{2}{|c|}{$/ \Xi_{c c}$} & \multicolumn{2}{c|}{$/ \Omega_{s c c}$} & \multicolumn{2}{c}{$/ \Omega_{c c c}$} \\
\cline { 2 - 7 } & RHIC & LHC & RHIC & LHC & RHIC & LHC \\
\hline$\Omega_{s c c}$ & 0.27 & 0.18 & & & & \\
$\Omega_{c c c}$ & 0.011 & 0.014 & 0.038 & 0.050 & & \\
$X_{4}$ & 0.056 & 0.039 & 0.20 & 0.21 & 5.3 & 4.3 \\
\hline \hline Coal. & \multicolumn{2}{|c|}{$/ \Xi_{c c}$} & \multicolumn{2}{|c|}{$/ \Omega_{s c c}$} & \multicolumn{2}{|c|}{$/ \Omega_{c c c}$} \\
\cline { 2 - 7 } & RHIC & LHC & RHIC & LHC & RHIC & LHC \\
\hline$\Omega_{s c c}$ & 0.29 & 0.18 & & & & \\
$\Omega_{c c c}$ & 0.00079 & 0.0011 & 0.0044 & 0.0059 & & \\
$X_{4}$ & 0.061 & 0.027 & 0.21 & 0.15 & 49 & 25 \\
\hline \hline
\end{tabular}

\section{TRANSVERSE MOMENTUM DISTRIBUTIONS OF MULTI-CHARMED HADRONS}

We consider in the coalescence model the transverse momentum distribution of multi-charmed hadrons, $\Xi_{c c}$, $\Xi_{c c}^{*}, \Omega_{s c c}, \Omega_{s c c}^{*}, \Omega_{c c c}$ baryons, $X(3872)$ and $T_{c c}$ mesons produced from one or two light and two or three charm quarks. Starting from the yield equation in the coalescence model [13] we obtain the transverse momentum distribution of the $\Xi_{c c}, \Xi_{c c}^{*}, \Omega_{s c c}, \Omega_{s c c}^{*}, \Omega_{c c c}$ baryon, the $X(3872)$ and $T_{c c}$ meson, respectively.

\section{A. Transverse momentum distributions of $\Xi_{c c}, \Xi_{c c}^{*}$,} $\Omega_{s c c}, \Omega_{s c c}^{*}$ and $\Omega_{c c c}$ baryons

The yield for the $\Xi_{c c}$ baryon produced from one light quark $l$ and two charm quarks, $c_{1}$ and $c_{2}$ is given by,

$$
\begin{aligned}
N_{\Xi_{c c}} & =g_{\Xi_{c c}} \int p_{l} \cdot d \sigma_{l} p_{c_{1}} \cdot d \sigma_{c_{1}} p_{c_{2}} \cdot d \sigma_{c_{2}} \frac{d^{3} \vec{p}_{l}}{(2 \pi)^{3} E_{l}} \\
& \times \frac{d^{3} \vec{p}_{c_{1}}}{(2 \pi)^{3} E_{c_{1}}} \frac{d^{3} \vec{p}_{c_{2}}}{(2 \pi)^{3} E_{c_{2}}} f_{l}\left(r_{l}, p_{l}\right) f_{c_{1}}\left(r_{c_{1}}, p_{c_{1}}\right) \\
& \times f_{c_{2}}\left(r_{c_{2}}, p_{c_{2}}\right) W_{\Xi_{c c}}\left(r_{l}, r_{c_{1}}, r_{c_{2}} ; p_{l}, p_{c_{1}}, p_{c_{2}}\right),
\end{aligned}
$$

with $d \sigma_{q}$ being the space-like hypersurface element for a quark $q . f_{q}\left(r_{q}, p_{q}\right)$ is a covariant distribution function of a quark $q$ satisfying the normalization condition $\int p_{q}$. $d \sigma_{q} d^{3} \vec{p}_{q} /\left((2 \pi)^{3} E\right) f_{q}\left(r_{q}, p_{q}\right)=N_{q}$, the number of quarks $q$ in the system. The factor $g_{\Xi_{c c}}$ takes into account the possibility of forming the $\Xi_{c c}$ baryon from constituent quarks, e.g., $g_{\Xi_{c c}}=2 \times 2 /(2 \cdot 3)^{3}$. In the non-relativistic limit, Eq. (2) is reduced to [12, 13, 38]

$$
\begin{aligned}
\frac{d^{2} N_{\Xi_{c c}}}{d^{2} \vec{p}_{T}} & =\frac{g_{\Xi_{c c}}}{V^{2}} \int d^{3} \vec{r}_{1} d^{3} \vec{r}_{2} d^{2} \vec{p}_{l T} d^{2} \vec{p}_{c_{1} T} d^{2} \vec{p}_{c_{2} T} \\
& \times \delta^{(2)}\left(\vec{p}_{T}-\vec{p}_{l T}-\vec{p}_{c_{1} T}-\vec{p}_{c_{2} T}\right) \frac{d^{2} N_{l}}{d^{2} \vec{p}_{l T}} \\
& \times \frac{d^{2} N_{c_{1}}}{d^{2} \vec{p}_{c_{1} T}} \frac{d^{2} N_{c_{2}}}{d^{2} \vec{p}_{c_{2} T}} W_{\Xi_{c c}}\left(\vec{r}_{1}, \vec{r}_{2}, \vec{r}_{3}, \vec{k}_{1}, \vec{k}_{2}, \vec{k}_{3}\right),(3)
\end{aligned}
$$

with the assumption of the boost-invariant longitudinal momentum distributions for quarks satisfying $\eta=y$, the Bjorken correlation between spatial, $\eta$ and momentum $y$ rapidities. In Eq. (3) $\vec{r}_{i}$ and $\vec{k}_{i}$ are relative distances and transverse momenta between quarks, respectively. $\vec{k}_{i}$ is related to the transverse momenta of quarks through the Lorentz transformation, from the transverse momenta in the rest frame of the produced $\Xi_{c c}$ baryon, $\vec{p}_{i T}$ to those in the fireball rest frame, $\vec{p}_{i T}^{\prime}$ [37, 38]. We consider here the following quark configuration which has been used in 
[17, 18],

$$
\begin{aligned}
& \vec{R}=\vec{r}_{l}+\vec{r}_{c_{1}}+\vec{r}_{c_{2}}, \\
& \vec{r}_{1}=\vec{r}_{c_{1}}-\vec{r}_{c_{2}}, \\
& \vec{r}_{2}=\frac{m_{c} \vec{r}_{c_{1}}+m_{c} \vec{r}_{c_{2}}}{m_{c}+m_{c}}-\vec{r}_{l},
\end{aligned}
$$

for relative quark coordinates, and

$$
\begin{aligned}
& \vec{k}=\vec{p}_{l T}^{\prime}+\vec{p}_{c_{1} T}^{\prime}+\vec{p}_{c_{2} T}^{\prime}, \\
& \vec{k}_{1}=\frac{m_{c} \vec{p}_{c_{1} T}^{\prime}-m_{c} \vec{p}_{c_{2} T}^{\prime}}{m_{c}+m_{c}}, \\
& \vec{k}_{2}=\frac{m_{l}\left(\vec{p}_{c_{1} T}^{\prime}+\vec{p}_{c_{2} T}^{\prime}\right)-\left(m_{c}+m_{c}\right) \vec{p}_{l T}^{\prime}}{m_{l}+m_{c}+m_{c}},
\end{aligned}
$$

for relative quark transverse momenta. Reduced masses corresponding to the above configurations are

$$
\mu_{1}=\frac{m_{c} m_{c}}{m_{c}+m_{c}}, \quad \mu_{2}=\frac{\left(m_{c}+m_{c}\right) m_{l}}{m_{l}+m_{c}+m_{c}} .
$$

We adopt the following $s$-wave Wigner function constructed from harmonic oscillator wave functions,

$$
\begin{aligned}
& W_{\Xi_{c c}}\left(\vec{r}_{1}, \vec{r}_{2}, \vec{k}_{1}, \vec{k}_{2}\right) \\
& \quad=8^{2} \exp \left(-\frac{r_{1}^{2}}{\sigma_{1}^{2}}-\sigma_{1}^{2} k_{1}^{2}\right) \exp \left(-\frac{r_{2}^{2}}{\sigma_{2}^{2}}-\sigma_{2}^{2} k_{2}^{2}\right)(7)
\end{aligned}
$$

with $\sigma_{i}^{2}=1 /\left(\mu_{i} \omega\right)$, where $\omega$ is the oscillator frequency of the harmonic oscillator wave function. After plugging the Wigner function, Eq. (7) into Eq. (3), and carrying out the coordinate space integration we obtain,

$$
\begin{aligned}
\frac{d^{2} N_{\Xi_{c c}}}{d^{2} \vec{p}_{T}} & =\frac{g_{\Xi_{c c}}}{V^{2}}(2 \sqrt{\pi})^{6}\left(\sigma_{1} \sigma_{2}\right)^{3} \int d^{2} \vec{p}_{l T} d^{2} \vec{p}_{c_{1} T} d^{2} \vec{p}_{c_{2} T} \\
& \times \delta^{(2)}\left(\vec{p}_{T}-\vec{p}_{l T}-\vec{p}_{c_{1} T}-\vec{p}_{c_{2} T}\right) \frac{d^{2} N_{l}}{d^{2} \vec{p}_{l T}} \\
& \times \frac{d^{2} N_{c_{1}}}{d^{2} \vec{p}_{c_{1} T}} \frac{d^{2} N_{c_{2}}}{d^{2} \vec{p}_{c_{2} T}} \exp \left(-\sigma_{1}^{2} k_{1}^{2}-\sigma_{2}^{2} k_{2}^{2}\right) .
\end{aligned}
$$

Similarly, the yield of the $\Xi_{c c}^{*}$ baryon produced from the same quark constituents as the $\Xi_{c c}$ baryon is given by,

$$
\begin{aligned}
\frac{d^{2} N_{\Xi_{c c}^{*}}}{d^{2} \vec{p}_{T}} & =\frac{g_{\Xi_{c c}^{*}}}{V^{2}}(2 \sqrt{\pi})^{6}\left(\sigma_{1} \sigma_{2}\right)^{3} \int d^{2} \vec{p}_{l T} d^{2} \vec{p}_{c_{1} T} d^{2} \vec{p}_{c_{2} T} \\
& \times \delta^{(2)}\left(\vec{p}_{T}-\vec{p}_{l T}-\vec{p}_{c_{1} T}-\vec{p}_{c_{2} T}\right) \frac{d^{2} N_{l}}{d^{2} \vec{p}_{l T}} \\
& \times \frac{d^{2} N_{c_{1}}}{d^{2} \vec{p}_{c_{1} T}} \frac{d^{2} N_{c_{2}}}{d^{2} \vec{p}_{c_{2} T}} \exp \left(-\sigma_{1}^{2} k_{1}^{2}-\sigma_{2}^{2} k_{2}^{2}\right) .
\end{aligned}
$$

with $g_{\Xi_{c c}^{*}}=2 \times 4 /(2 \cdot 3)^{3}$ being the chance of forming $\Xi_{c c}^{*}$ baryon from one light and two charm quarks. The yield of the $\Omega_{s c c}$ baryon produced from one strange, $s$ and two charm quarks, $c_{1}, c_{2}$ is then given by,

$$
\begin{gathered}
\frac{d^{2} N_{\Omega_{s c c}}}{d^{2} \vec{p}_{T}}=\frac{g_{\Omega_{s c c}}}{V^{2}}(2 \sqrt{\pi})^{6}\left(\sigma_{1} \sigma_{2}\right)^{3} \int d^{2} \vec{p}_{c_{1} T} d^{2} \vec{p}_{c_{2} T} d^{2} \vec{p}_{s T} \\
\quad \times \delta^{(2)}\left(\vec{p}_{T}-\vec{p}_{c_{1} T}-\vec{p}_{c_{2} T}-\vec{p}_{s T}\right) \frac{d^{2} N_{c_{1}}}{d^{2} \vec{p}_{c_{1} T}} \\
\quad \times \frac{d^{2} N_{c_{2}}}{d^{2} \vec{p}_{c_{2} T}} \frac{d^{2} N_{s}}{d^{2} \vec{p}_{s T}} \exp \left(-\sigma_{1}^{2} k_{1}^{2}-\sigma_{2}^{2} k_{2}^{2}\right),
\end{gathered}
$$

with $g_{\Omega_{s c c}}=2 /(2 \cdot 3)^{3}$ being the chance of forming $\Omega_{s c c}$ baryon from one strange and two charm quarks. Also similarly, the yield of the $\Omega_{s c c}^{*}$ baryon produced from the same constituents as the $\Omega_{s c c}$ is given by,

$$
\begin{gathered}
\frac{d^{2} N_{\Omega_{s c c}^{*}}}{d^{2} \vec{p}_{T}}=\frac{g_{\Omega_{s c c}^{*}}}{V^{2}}(2 \sqrt{\pi})^{6}\left(\sigma_{1} \sigma_{2}\right)^{3} \int d^{2} \vec{p}_{c_{1} T} d^{2} \vec{p}_{c_{2} T} d^{2} \vec{p}_{s T} \\
\quad \times \delta^{(2)}\left(\vec{p}_{T}-\vec{p}_{c_{1} T}-\vec{p}_{c_{2} T}-\vec{p}_{s T}\right) \frac{d^{2} N_{c_{1}}}{d^{2} \vec{p}_{c_{1} T}} \\
\quad \times \frac{d^{2} N_{c_{2}}}{d^{2} \vec{p}_{c_{2} T}} \frac{d^{2} N_{s}}{d^{2} \vec{p}_{s T}} \exp \left(-\sigma_{1}^{2} k_{1}^{2}-\sigma_{2}^{2} k_{2}^{2}\right),
\end{gathered}
$$

with the chance of forming $\Omega_{s c c}^{*}$ baryon from one strange and two charm quarks, $g_{\Omega_{s c c}^{*}}=4 /(2 \cdot 3)^{3}$. Finally the yield of the $\Omega_{c c c}$ baryon produced from three charm quarks, $c_{1}$, $c_{2}$ and $c_{3}$ is,

$$
\begin{aligned}
& \frac{d^{2} N_{\Omega_{c c c}}}{d^{2} \vec{p}_{T}}=\frac{g_{\Omega_{c c c}}}{V^{2}}(2 \sqrt{\pi})^{6}\left(\sigma_{1} \sigma_{2}\right)^{3} \int d^{2} \vec{p}_{c_{1} T} d^{2} \vec{p}_{c_{2} T} d^{2} \vec{p}_{c_{3} T} \\
& \quad \times \delta^{(2)}\left(\vec{p}_{T}-\vec{p}_{c_{1} T}-\vec{p}_{c_{2} T}-\vec{p}_{c_{3} T}\right) \frac{d^{2} N_{c_{1}}}{d^{2} \vec{p}_{c_{1} T}} \\
& \quad \times \frac{d^{2} N_{c_{2}}}{d^{2} \vec{p}_{c_{2} T}} \frac{d^{2} N_{c_{3}}}{d^{2} \vec{p}_{c_{3} T}} \exp \left(-\sigma_{1}^{2} k_{1}^{2}-\sigma_{2}^{2} k_{2}^{2}\right)
\end{aligned}
$$

with $g_{\Omega_{c c c}}=4 /(2 \cdot 3)^{3}$ being the chance of forming $\Omega_{c c c}$ baryon from three charm quarks.

\section{B. Transverse momentum distributions of the $X(3872)$ and $T_{c c}$ meson}

We also start with the yield equation in the coalescence model in order to construct the transverse momentum distribution of the $X(3872)$ meson produced from two light quarks $l, \bar{l}$ and two charm quarks, $c$ and $\bar{c}$,

$$
\begin{aligned}
N_{X}= & g_{X} \int p_{l} \cdot d \sigma_{l} p_{\bar{l}} \cdot d \sigma_{\bar{l}} p_{c} \cdot d \sigma_{c} p_{\bar{c}} \cdot d \sigma_{\bar{c}} \\
& \times \frac{d^{3} \vec{p}_{l}}{(2 \pi)^{3} E_{l}} \frac{d^{3} \vec{p}_{\bar{l}}}{(2 \pi)^{3} E_{\bar{l}}} \frac{d^{3} \vec{p}_{c}}{(2 \pi)^{3} E_{c}} \frac{d^{3} \vec{p}_{\bar{c}}}{(2 \pi)^{3} E_{\bar{c}}} \\
& \times f_{l}\left(r_{l}, p_{l}\right) f_{\bar{l}}\left(r_{\bar{l}}, p_{\bar{l}}\right) f_{c}\left(r_{c}, p_{c}\right) f_{\bar{c}}\left(r_{\bar{c}}, p_{\bar{c}}\right) \\
& \times W_{X}\left(r_{l}, r_{\bar{l}}, r_{c}, r_{\bar{c}} ; p_{l}, p_{\bar{l}}, p_{c}, p_{\bar{c}}\right),
\end{aligned}
$$

with the same configurations introduced in Eq. (22); $d \sigma_{q}, f_{q}\left(r_{q}, p_{q}\right)$ are, respectively the space-like hypersurface element for a quark $q$, and a quark $q$ covariant distribution function with the normalization $\int p_{q}$. 
$d \sigma_{q} d^{3} \vec{p}_{q} /\left((2 \pi)^{3} E\right) f_{q}\left(r_{q}, p_{q}\right)=N_{q}$, the number of $q$ quarks in the system. The factor $g_{X}$ covers the possibility of forming the $X(3872)$ meson from constituent quarks, e.g., $g_{X}=3 /(2 \cdot 3)^{4}$. Eq. (13) is reduced in the non-relativistic limit to [12, 13, 38]

$$
\begin{aligned}
\frac{d^{2} N_{X}}{d^{2} \vec{p}_{T}} & =\frac{g_{X}}{V^{3}} \int d^{3} \vec{r}_{1} d^{3} \vec{r}_{2} d^{3} \vec{r}_{3} d^{2} \vec{p}_{l T} d^{2} \vec{p}_{\bar{l} T} d^{2} \vec{p}_{c T} d^{2} \vec{p}_{\bar{c} T} \\
& \times \delta^{(2)}\left(\vec{p}_{T}-\vec{p}_{l T}-\vec{p}_{\bar{l} T}-\vec{p}_{c T}-\vec{p}_{\bar{c} T}\right) \frac{d^{2} N_{l}}{d^{2} \vec{p}_{l T}} \frac{d^{2} N_{\bar{l}}}{d^{2} \vec{p}_{\bar{l} T}} \\
& \times \frac{d^{2} N_{c}}{d^{2} \vec{p}_{c T}} \frac{d^{2} N_{\bar{c}}}{d^{2} \vec{p}_{\bar{c} T}} W_{X}\left(\vec{r}_{1}, \vec{r}_{2}, \vec{r}_{3}, \vec{k}_{1}, \vec{k}_{2}, \vec{k}_{3}\right),
\end{aligned}
$$

again with the boost-invariant longitudinal momentum distribution assumption for quarks; $\eta=y$, the Bjorken correlation between spatial, $\eta$ and momentum $y$ rapidities. In Eq. (14) relative transverse momenta, $\vec{k}_{i}$ are related to the transverse momenta of quarks $\vec{p}_{i T}$ through the Lorentz transformation from the transverse momenta in the rest frame of the produced $X(3872)$ meson, $\vec{p}_{i T}$ to those in the fireball rest frame, $\vec{p}_{i T}^{\prime}[37,38]$. The configuration of quarks inside the $X(3872)$ meson is not unique, and possible quark arrangements for the $X(3872)$ meson are shown in the Appendix A. We adopt here the following quark configuration for the $X(3872)$ meson,

$$
\begin{aligned}
& \vec{R}=\vec{r}_{l}+\vec{r}_{\bar{l}}+\vec{r}_{c}+\vec{r}_{\bar{c}}, \\
& \vec{r}_{1}=\vec{r}_{l}-\vec{r}_{\bar{l}}, \\
& \vec{r}_{2}=\frac{m_{l} \vec{r}_{l}+m_{\bar{l}} \vec{r}_{\bar{l}}}{m_{l}+m_{\bar{l}}}-\vec{r}_{c}, \\
& \vec{r}_{3}=\frac{m_{l} \vec{r}_{l}+m_{\bar{l}} \vec{r}_{\bar{l}}+m_{c} \vec{r}_{c}}{m_{l}+m_{\bar{l}}+m_{c}}-\vec{r}_{\bar{c}},
\end{aligned}
$$

for relative quark coordinates, and

$$
\begin{aligned}
& \vec{k}=\vec{p}_{l T}^{\prime}+\vec{p}_{\bar{l} T}^{\prime}+\vec{p}_{c T}^{\prime}+\vec{p}_{\bar{c} T}^{\prime}, \\
& \vec{k}_{1}=\frac{m_{\bar{l}} \vec{p}_{l T}^{\prime}-m_{l} \vec{p}_{\bar{l} T}^{\prime}}{m_{l}+m_{\bar{l}}}, \\
& \vec{k}_{2}=\frac{m_{c}\left(\vec{p}_{l T}^{\prime}+\vec{p}_{\bar{l} T}^{\prime}\right)-\left(m_{l}+m_{\bar{l}}\right) \vec{p}_{c T}^{\prime}}{m_{l}+m_{\bar{l}}+m_{c}}, \\
& \left.\vec{k}_{3}=\frac{m_{\bar{c}}\left(\vec{p}_{l T}^{\prime}+\vec{p}_{\bar{l} T}^{\prime}+\vec{p}_{c T}^{\prime}\right)-\left(m_{l}+m_{\bar{l}}+m_{c}\right) \vec{p}_{\bar{c} T}^{\prime}}{m_{l}+m_{\bar{l}}+m_{c}+m_{\bar{c}}}, 16\right)
\end{aligned}
$$

for relative quark transverse momenta. Then, reduced masses corresponding to the above configurations becomes,

$$
\begin{aligned}
\mu_{1} & =\frac{m_{l} m_{\bar{l}}}{m_{l}+m_{\bar{l}}}, \quad \mu_{2}=\frac{\left(m_{l}+m_{\bar{l}}\right) m_{c}}{m_{l}+m_{\bar{l}}+m_{c}}, \\
\mu_{3} & =\frac{\left(m_{l}+m_{\bar{l}}+m_{c}\right) m_{\bar{c}}}{m_{l}+m_{\bar{l}}+m_{c}+m_{\bar{c}}} .
\end{aligned}
$$

With the $s$-wave Wigner function made up of harmonic oscillator wave functions,

$$
\begin{aligned}
& W_{X}\left(\vec{r}_{1}, \vec{r}_{2}, \vec{r}_{3}, \vec{k}_{1}, \vec{k}_{2}, \vec{k}_{3}\right) \\
& \quad=8^{3} \exp \left(-\frac{r_{1}^{2}}{\sigma_{1}^{2}}-\sigma_{1}^{2} k_{1}^{2}\right) \exp \left(-\frac{r_{2}^{2}}{\sigma_{2}^{2}}-\sigma_{2}^{2} k_{2}^{2}\right) \\
& \quad \times \exp \left(-\frac{r_{3}^{2}}{\sigma_{3}^{2}}-\sigma_{3}^{2} k_{3}^{2}\right)
\end{aligned}
$$

we obtain the transverse momentum distribution of the $X(3872)$ meson,

$$
\begin{aligned}
\frac{d^{2} N_{X}}{d^{2} \vec{p}_{T}} & =\frac{g_{X}}{V^{3}}(2 \sqrt{\pi})^{9}\left(\sigma_{1} \sigma_{2} \sigma_{3}\right)^{3} \int d^{2} \vec{p}_{l T} d^{2} \vec{p}_{\bar{l} T} d^{2} \vec{p}_{c T} d^{2} \vec{p}_{\bar{c} T} \\
& \times \delta^{(2)}\left(\vec{p}_{T}-\vec{p}_{l T}-\vec{p}_{\bar{l} T}-\vec{p}_{c T}-\vec{p}_{\bar{c} T}\right) \frac{d^{2} N_{l}}{d^{2} \vec{p}_{l T}} \frac{d^{2} N_{\bar{l}}}{d^{2} \vec{p}_{\bar{l} T}} \\
& \times \frac{d^{2} N_{c}}{d^{2} \vec{p}_{c T}} \frac{d^{2} N_{\bar{c}}}{d^{2} \vec{p}_{\bar{c} T}} \exp \left(-\sigma_{1}^{2} k_{1}^{2}-\sigma_{2}^{2} k_{2}^{2}-\sigma_{3}^{2} k_{3}^{2}\right)(19)
\end{aligned}
$$

On the other hand, the transverse momentum distribution of the $X(3872)$ meson produced from a charm and an anti-charm quark is given by,

$$
\begin{aligned}
\frac{d^{2} N_{X_{2}}}{d^{2} \vec{p}_{T}} & =\frac{g_{X}}{V}(2 \sqrt{\pi})^{3} \sigma^{3} \int d^{2} \vec{p}_{c T} d^{2} \vec{p}_{\bar{c} T} \frac{2}{3} \sigma^{2} k^{2} e^{-\sigma^{2} k^{2}} \\
& \times \delta^{(2)}\left(\vec{p}_{T}-\vec{p}_{c T}-\vec{p}_{\bar{c} T}\right) \frac{d^{2} N_{c}}{d^{2} \vec{p}_{c T}} \frac{d^{2} N_{\bar{c}}}{d^{2} \vec{p}_{\bar{c} T}}
\end{aligned}
$$

which is same in form as the transverse momentum distribution of the $p$-wave charmonium state $\chi_{c}$ [30]. In Eq. (20), $\vec{k}=\left(m_{\bar{c}} \vec{p}_{c T}^{\prime}-m_{c} \vec{p}_{\bar{c} T}^{\prime}\right) /\left(m_{c}+m_{\bar{c}}\right)$ and $\sigma^{2}=1 / \mu \omega=$ $\left(m_{c}+m_{\bar{c}}\right) / m_{c} / m_{\bar{c}} / \omega$. The transverse momentum distribution of the $T_{c c}$ is similar to that of the $X(3872)$ composed of four quarks,

$$
\begin{aligned}
\frac{d^{2} N_{T_{c c}}}{d^{2} \vec{p}_{T}} & =\frac{g_{T_{c c}}}{V^{3}}(2 \sqrt{\pi})^{9}\left(\sigma_{1} \sigma_{2} \sigma_{3}\right)^{3} \int d^{2} \vec{p}_{\bar{l}_{1} T} d^{2} \vec{p}_{\bar{l}_{2} T} d^{2} \vec{p}_{c_{1} T} \\
& \times d^{2} \vec{p}_{c_{2} T} \delta^{(2)}\left(\vec{p}_{T}-\vec{p}_{\bar{l}_{1} T}-\vec{p}_{\bar{l}_{2} T}-\vec{p}_{c_{1} T}-\vec{p}_{c_{2} T}\right) \\
& \times \frac{d^{2} N_{\bar{l}_{1}}}{d^{2} \vec{p}_{\bar{l}_{1} T}} \frac{d^{2} N_{\bar{l}_{2}}}{d^{2} \vec{p}_{\bar{l}_{2} T}} \frac{d^{2} N_{c_{1}}}{d^{2} \vec{p}_{c_{1} T}} \frac{d^{2} N_{c_{2}}}{d^{2} \vec{p}_{c_{2} T}} \\
& \times \exp \left(-\sigma_{1}^{2} k_{1}^{2}-\sigma_{2}^{2} k_{2}^{2}-\sigma_{3}^{2} k_{3}^{2}\right)
\end{aligned}
$$

with $g_{T_{c c}}=3 /(2 \cdot 3)^{4}$ being the chance of forming the $T_{c c}$ meson from two light and two charm quarks.

\section{Charm and light quark transverse momentum distributions}

In order to evaluate the transverse momentum distribution of a multi-charmed hadron we need the information on the transverse momentum distribution of both light and charm quarks in the system. We introduce here the following transverse momentum distributions at mid-rapidities [39], 


$$
\begin{aligned}
& \frac{d^{2} N_{c}^{R}}{d^{2} \vec{p}_{c T}}= \begin{cases}0.69 e^{\left(-1.22 p_{c T}^{1.57}\right)} & p_{c T} \leq 1.85 \mathrm{GeV} \\
1.08 e^{\left(-3.04 p_{c T}^{0.71}\right)}+3.79\left(1.0+p_{c T}^{2.02}\right)^{-3.48} & p_{c T}>1.85 \mathrm{GeV}\end{cases} \\
& \frac{d^{2} N_{c}^{L}}{d^{2} \vec{p}_{c T}}= \begin{cases}1.97 e^{\left(-0.35 p_{c T}^{2.47}\right)} & p_{c T} \leq 1.85 \mathrm{GeV} \\
7.95 e^{\left(-3.49 p_{c T}^{3.59}\right)}+87335\left(1.0+p_{c T}^{0.5}\right)^{-14.31} & p_{c T}>1.85 \mathrm{GeV}\end{cases}
\end{aligned}
$$

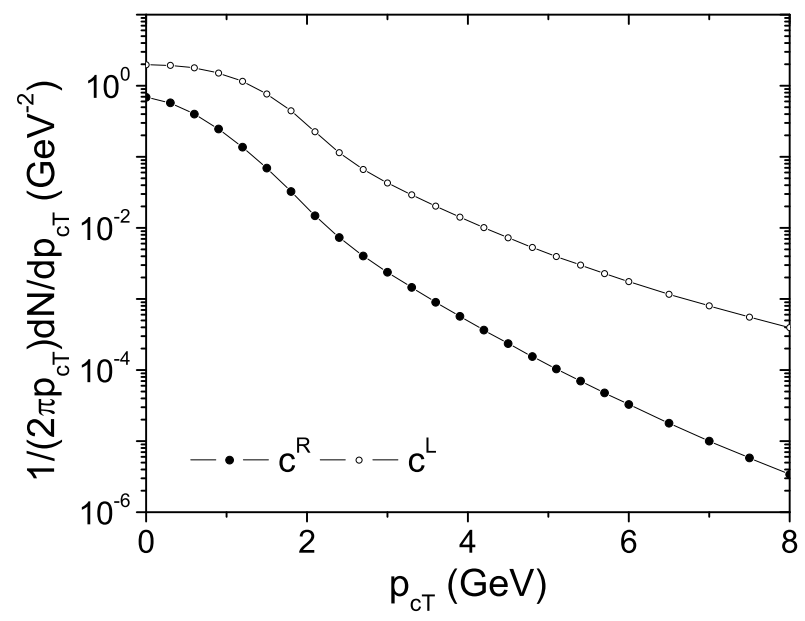

FIG. 1: (a) Transverse momentum distributions of charm quarks $1 /\left(2 \pi p_{c T}\right) d N / d p_{c T}$ at RHIC and LHC.

for charm quarks shown in Fig. 1 $d^{2} N_{q} / d^{2} \vec{p}_{q T}$ with a superscript $R$ and $L$ in Eq. (22) represents a charm quark transverse momentum distribution at RHIC and LHC, respectively.

For the light quark transverse momentum distribution we adopt the following thermal distribution,

$$
\frac{d^{2} N_{l}}{d^{2} p_{T}}=g_{l} \frac{V}{(2 \pi)^{3}} m_{T} e^{-m_{T} / T_{e f f}},
$$

with the color-spin degeneracy of light quarks, $g_{l}=6$ and the transverse mass $m_{T}=\sqrt{p_{T}^{2}+m^{2}}$. We use the same coalescence volume 1790 and $3530 \mathrm{fm}^{3}$ for RHIC and LHC, respectively introduced in Ref. [19]. $T_{\text {eff }}$ in Eq. (23) is an effective temperature which we take here as $T_{\text {eff }}=177 \mathrm{MeV}$ for both RHIC and LHC in order to take into account collective flow effects of the quark-gluon plasma. Even though the same effective temperature is applied for both RHIC and LHC, the larger flow effects at LHC have been taken into account since freeze-out temperatures estimated in the statistical hadronization model are 162 and $156 \mathrm{MeV}$ at RHIC and LHC.

Light quarks also have a power law type transverse momentum distribution at high transverse momentum. However, for such effects to be visible in a charmed hadron, the momentum of a charm quark or a charmed hadron itself should have an even larger transverse momentum because the transverse momentum of heavy quark hadrons is mostly dominated by the momentum of the heavy quarks. Therefore, it is reasonable to consider only light quarks with an exponential transverse momentum distribution when we are interested in the transverse momentum distribution of a heavy quark hadron up to about $8 \mathrm{GeV}$.

Using the above transverse momentum distributions with the light quark mass $300 \mathrm{MeV}$, we obtain the total number of charm quarks available in the system, $N_{c}=$ 2.00 at RHIC and $N_{c}=14.9$ at LHC, and also the total number of light quarks $N_{l}=298$ at RHIC and $N_{l}=588$ at LHC comparable to those in Ref. [19].

Finally, we have to determine the oscillator frequency, $\omega_{c}$. Since the oscillator frequency is related to the size of the hadron in the coalescence model, it is mostly determined from the relation between the mean square distance $\left\langle r^{2}\right\rangle$ and $\sigma$; for a $D$ meson $\left\langle r^{2}\right\rangle=3 / 2 \sigma^{2}=$ $3 / 2 / \mu / \omega_{c}$. However, we take here the oscillator frequency which enables all charm quarks at zero transverse momentum to get hadronized entirely by quark coalescence [38, 39]. It has been found that hadrons are produced via two different hadron production mechanisms in heavy ion collisions, one by quark coalescence or the other by fragmentation 12 15. Since the hadron production by quark coalescence is dominant at low transverse momenta, it is natural to expect exclusive hadron production at zero transverse momentum by quark coalescence.

In extracting those oscillator frequencies we consider the transverse momentum distributions for four open charm mesons, $D, D^{*}, D_{s}$, and $D_{s}^{*}$, and ten charm baryons, $\Lambda_{c}, \Sigma_{c}(2455), \Sigma_{c}(2520), \Lambda_{c}(2595), \Lambda_{c}(2625), \Xi_{c}$, $\Xi_{c}^{\prime}, \Xi_{c}(2645), \Omega_{c}$, and $\Omega_{c}(2770)$. Charmonium states and multi-charmed hadrons are not taken into account in the evaluation of the oscillator frequency since the transverse momentum distributions of charmonium states or multicharmed hadrons are negligible compared to those of charmed hadrons mentioned above. We find that oscillator frequencies, $\omega_{c}=0.078 \mathrm{GeV}$ at RHIC and $\omega_{c}=0.076$ $\mathrm{GeV}$ at LHC guarantee the consumption of all charm quarks at zero transverse momentum entirely by quark coalescence.

The oscillator frequencies $\omega_{c}=0.078$ or $0.076 \mathrm{GeV}$ are smaller than those in Ref. [19], $\omega_{c}=0.244$ or $0.276 \mathrm{GeV}$ obtained on two conditions; the sum of yields for charmed hadrons, e.g., $D, D^{*}$, and $D_{s}$ mesons and $\Lambda_{c}$ baryons after hadronization in the statistical hadronization model is 
equal to the total number of charm quarks available in the quark-gluon plasma before hadronization, and the yield of $\Lambda_{c}$ including the feed-down contributions in the coalescence model agrees with that in the statistical model. As has already been pointed out in Refs. 38, 39] smaller oscillator frequencies obtained on the requirement that all charm quarks at zero transverse momentum should be used up entirely by quark coalescence give the size of charmed hadrons relatively larger than the real size of those hadrons. When the charm quark oscillator frequencies $\omega_{c}=0.078$ or $0.076 \mathrm{GeV}$ are applied, the size of the $D$ meson, $\sqrt{\left\langle r^{2}\right\rangle}=1.73 \mathrm{fm}$, also larger than the assumed size of $D$ mesons, $\sim 1.0 \mathrm{fm}$.

With these charm quark oscillator frequencies we evaluate the transverse momentum distributions of $D^{0}$ mesons, and compare those with experimental measurements at RHIC [40] and LHC [41]. We take into account here the feed-down contribution from $D^{*}$ mesons as well as that from the production of $D$ mesons by fragmentation. We show results in Fig. 2

As we see in Fig. 2, transverse momentum distributions of $D$ mesons evaluated here in the coalescence model based on the charm and light quark transverse momentum distributions, Eqs. (22) and (23) with charm quark oscillator frequencies $\omega_{c}=0.078$ or $0.076 \mathrm{GeV}$ agree reasonably well with those measurements at RHIC and LHC. The transverse momentum distribution of $D^{0}$ mesons at RHIC is slightly smaller than the experimental measurement at intermediate transverse momenta whereas that at LHC deviates from measurements within errors at high transverse momenta.

\section{Transverse momentum distributions of multi-charmed hadrons}

Now we evaluate transverse momentum distributions of $\Xi_{c c}, \Xi_{c c}^{*}, \Omega_{s c c}, \Omega_{s c c}^{*}, \Omega_{c c c}$ baryons, $X(3872)$, and $T_{c c}$ mesons produced by quark recombination using Eqs. (8), (9), (10), (11), (12), (19), (20) and (21). In the calculation we use light quark mass $m_{l}=m_{\bar{l}}=300 \mathrm{MeV}$, charm quark mass $m_{c}=m_{\bar{c}}=1500 \mathrm{MeV}$, and the volume 1790 $\mathrm{fm}^{3}$ for RHIC and $3530 \mathrm{fm}^{3}$ for LHC.

We show in Fig. 3 transverse momentum distributions, $\left(2 \pi p_{T}\right)^{-1} d N / d p_{T}$ of multi-charmed hadrons, $\Xi_{c c}$, $\Xi_{c c}^{*}, \Omega_{s c c}, \Omega_{s c c}^{*}, \Omega_{c c c}$ baryons, and the $X(3872)$ meson in a four-quark state, $X_{4}$ at both RHIC $\sqrt{s_{N N}}=200 \mathrm{GeV}$ and LHC $\sqrt{s_{N N}}=2.76 \mathrm{TeV}$. Here we have taken into account feed-down contributions for transverse momentum distributions of the $\Xi_{c c}$ and $\Omega_{s c c}$ baryon from their spin $3 / 2$ hadrons, $\Xi_{c c}^{*}$ and $\Omega_{s c c}^{*}$ baryons, respectively. However, we assume that transverse momentum distributions of the daughter hadrons, $\Xi_{c c}$ and $\Omega_{s c c}$ baryons are almost same as those of $\Xi_{c c}^{*}$ and $\Omega_{s c c}^{*}$ baryons after the $\Xi_{c c}^{*}$ and $\Omega_{s c c}^{*}$ baryon decays to the $\Xi_{c c}$ and $\Omega_{s c c}$ baryon, respectively since $\Xi_{c c}$ and $\Omega_{s c c}$ baryons are much heavier than the other daughter hadron in the decay process.

As we see in Fig. 3, all transverse momentum distribu-
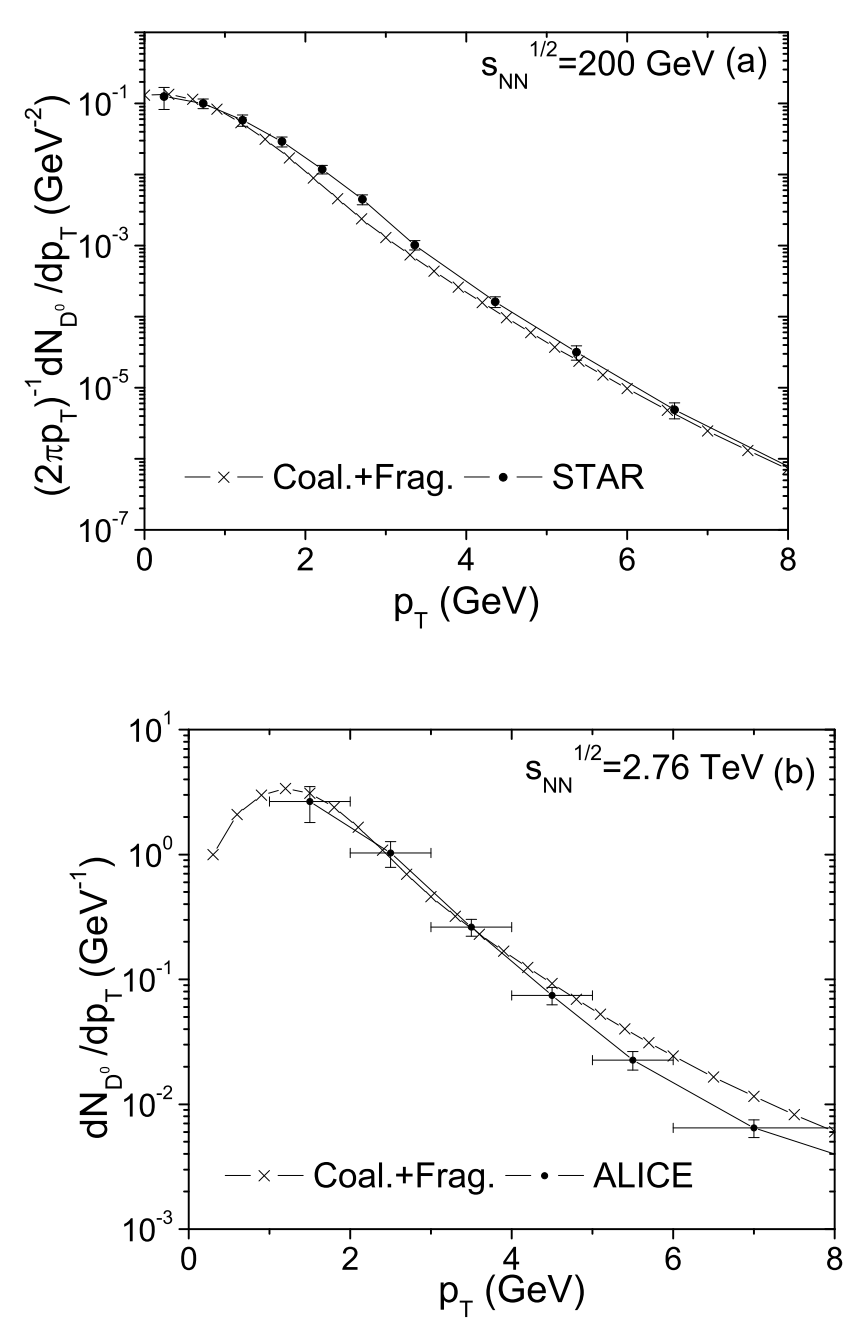

FIG. 2: (a) Transverse momentum distributions of $D^{0}$ mesons, $\left(2 \pi p_{T}\right)^{-1} d N_{D} / d p_{T}$ at RHIC and (b) those multiplied by $2 \pi p_{T}, d N_{D} / d p_{T}$ at LHC.

tions of charmed hadrons at LHC are larger than those at RHIC due to the larger number of charm and light quarks available at LHC compared to that at RHIC. It has been found that the yield of a hadron with more quarks is suppressed since the probability to combine more quarks to form a multi-quark hadron decreases as the number of quarks within a hadron is increased [17 19]. Therefore it is expected that the transverse momentum distribution of normal hadrons composed of three quarks, $\Xi_{c c}, \Xi_{c c}^{*}$, $\Omega_{s c c}$, and $\Omega_{s c c}^{*}$ baryons is larger than that of four-quark hadrons, $X(3872)$ mesons at both RHIC and LHC. We find that transverse momentum distributions of the $\Xi_{c c}$ and $\Xi_{c c}^{*}$ baryon is larger than that of the $X_{4}$ meson by an order of magnitude at both RHIC and LHC as expected but the transverse momentum distribution of the $X_{4}$ meson is larger than that of the $\Omega_{c c c}$ baryon by two orders of magnitude at both RHIC and LHC. The effect 

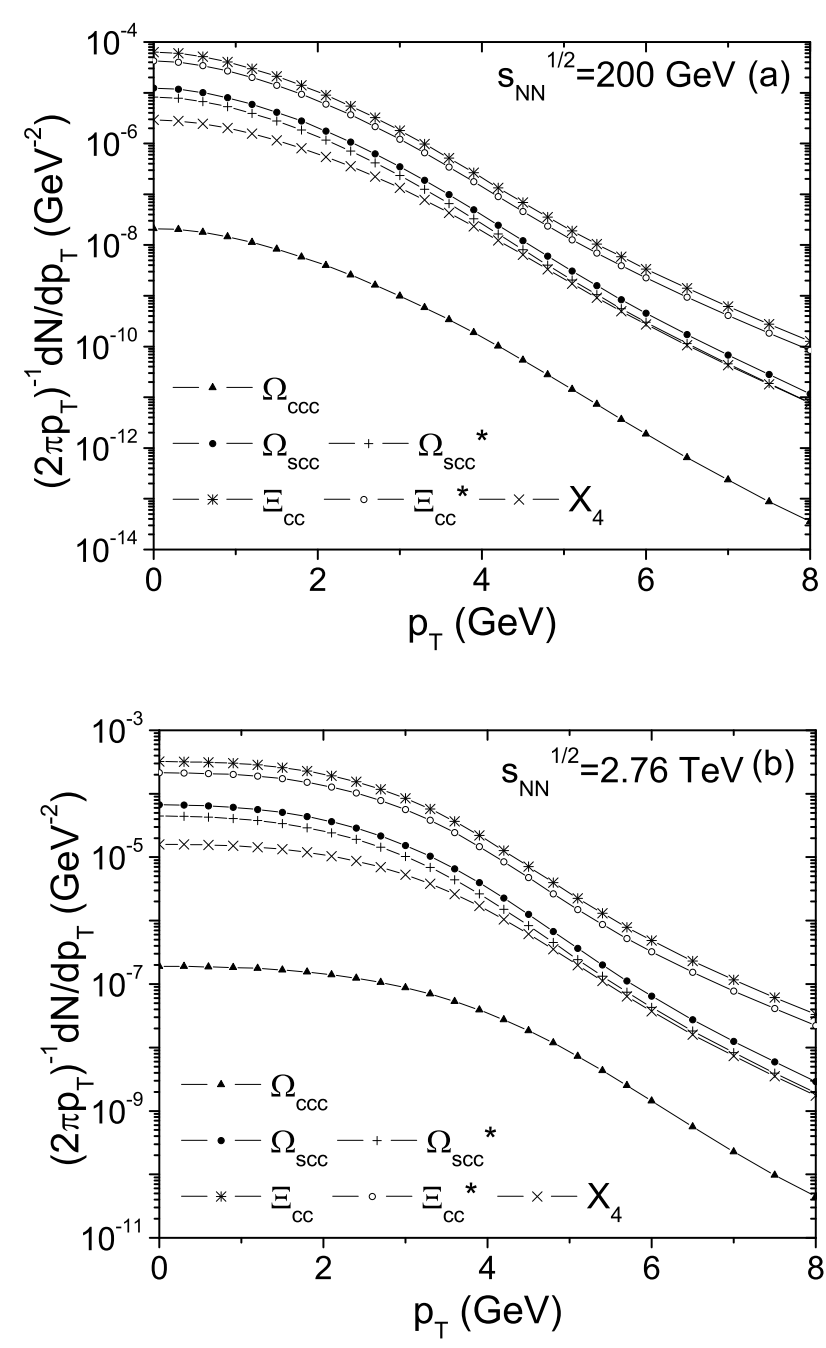

FIG. 3: Transverse momentum distributions, $\left(2 \pi p_{T}\right)^{-1} d N / d p_{T}$ of multi-charmed hadrons, $\Xi_{c c}, \Xi_{c c}^{*}$, $\Omega_{s c c}, \Omega_{s c c}^{*}, \Omega_{c c c}$ baryons, and a $X(3872)$ meson in a four-quark state, $X_{4}$ at RHIC (a) and at LHC (b).

from the much smaller abundance of charm quarks in the system compared to that of light quarks by a factor of hundreds at RHIC and LHC overwhelms the meaningful contribution from the larger possibility for forming a hadron composed of three charm quarks compared to the relatively smaller possibility for forming a hadron with four quarks, leading to the smaller transverse momentum distribution of the $\Omega_{c c c}$ baryon compared to that of the $X(3872)$ meson.

We have also obtained the transverse momentum distribution of the $T_{c c}$ meson, similar to that of the fourquark $X(3872)$ meson. Since the same transverse momentum distributions of charm quarks at RHIC and LHC, Eq. (22) are introduced, the difference of the transverse momentum distribution between the $T_{c c}$ and the $X_{4}$ is originated from the light quark/anti-quark distri-

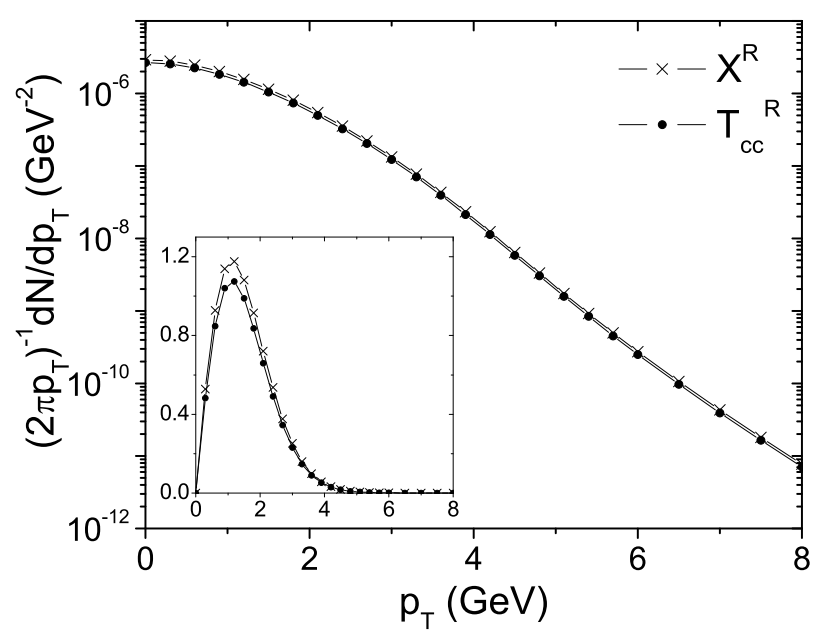

FIG. 4: Transverse momentum distributions of the $X(3872)$ in a four-quark state, $X_{4}$ and the $T_{c c}$ meson for $\sqrt{s_{N N}}=200$ $\mathrm{GeV}$ at RHIC. In the inset $d N / d p_{T}$ of the $X_{4}$ and $T_{c c}$ in unit of $10^{-5} \mathrm{GeV}^{-1}$ are shown.

butions, or the non-zero baryon chemical potential. We show in Fig. 4 the transverse momentum distributions of $X_{4}$ and $T_{c c}$ mesons at RHIC. As expected the transverse momentum distribution of the $T_{c c}$ is slightly smaller than that of the $X_{4}$ due to the smaller number of antilight quarks caused by the baryon chemical potential, $\mu_{l}=24 \mathrm{MeV}$ 32]. We do not show the transverse momentum distribution of the $T_{c c}$ at LHC since there exists no difference in the transverse momentum distributions between $X_{4}$ and $T_{c c}$ mesons with the zero baryon chemical potential.

TABLE IV: Total yields of the $\Xi_{c c}, \Xi_{c c}^{*}, \Omega_{s c c}, \Omega_{s c c}^{*}, \Omega_{c c c}$ baryon, the $T_{c c}$ and $X(3872)$ meson at mid-rapidity obtained by integrating the transverse momentum distributions shown in Fig. 3 over all transverse momenta at RHIC in $\sqrt{s_{N N}}=$ $200 \mathrm{GeV} \mathrm{Au}+\mathrm{Au}$ collisions and at LHC in $\sqrt{s_{N N}}=2.76 \mathrm{TeV}$ $\mathrm{Pb}+\mathrm{Pb}$ collisions.

\begin{tabular}{c|c|c}
\hline \hline & RHIC & LHC \\
\hline$\Xi_{c c}$ & $4.4 \times 10^{-4}$ & $6.7 \times 10^{-3}$ \\
$\Xi_{c c}^{*}$ & $2.9 \times 10^{-4}$ & $4.5 \times 10^{-3}$ \\
$\Omega_{s c c}$ & $8.6 \times 10^{-5}$ & $1.3 \times 10^{-3}$ \\
$\Omega_{s c c}^{*}$ & $5.7 \times 10^{-5}$ & $8.5 \times 10^{-4}$ \\
$\Omega_{c c c}$ & $1.7 \times 10^{-7}$ & $5.9 \times 10^{-6}$ \\
$T_{c c}$ & $2.2 \times 10^{-5}$ & $3.8 \times 10^{-4}$ \\
$X_{4}$ & $2.4 \times 10^{-5}$ & $3.8 \times 10^{-4}$ \\
$X_{2}$ & $2.6 \times 10^{-4}$ & $4.5 \times 10^{-3}$ \\
\hline \hline
\end{tabular}

We calculate the total yield of each charmed hadron by integrating out transverse momentum distributions 
shown in Fig. 3 over all transverse momenta, and summarize the results in Table IV. Here we have also taken into account feed-down contributions for the $\Xi_{c c}$ and $\Omega_{s c c}$ baryon from their spin $3 / 2$ hadrons, $\Xi_{c c}^{*}$ and $\Omega_{s c c}^{*}$ baryons, respectively. As we see in Table IV] the yields of the four-quark $X(3872)$ and the $T_{c c}$ at RHIC are now slightly different. We note that the yield of the $\Omega_{s c c}$ is slightly larger than that of the $X_{4}$. The yields based on the transverse momentum distributions are smaller and larger compared to those yields in Table @ at RHIC and LHC, respectively, and this difference must be mostly due to different numbers of charm quarks introduced in the system, 2.0 vs. 4.1 at RHIC and 14.9 vs. 11.0 at LHC.

\section{TRANSVERSE MOMENTUM DISTRIBUTION RATIOS}

\section{A. Transverse momentum distribution ratios between multi-charmed hadrons}

Based on transverse momentum distributions of multicharmed hadrons as shown in Fig. 3 we evaluate various transverse momentum distribution ratios between multi-charmed hadrons, similar to the anti-proton/pion ratio which shows an increase up to unity in the intermediate transverse momenta due to the contribution from two hadron production processes, the recombination and fragmentation [12 15]. The transverse momentum distribution ratio between an anti-proton and a pion is the ratio between a three-light quark and a two-light quark hadrons, $\bar{q} \bar{q} / q \bar{q}$ whereas the ratio evaluated here is, for example, the ratio between a two-charm and two-light quark and a two-charm and one-light quark hadrons, $c c \bar{q} \bar{q} / c c q$, or $c \bar{c} q \bar{q} / c c q, X(3872) / \Xi_{c c}$ and so on. After dividing out the same flavors appearing in the numerator and denominator, since both ratios simply retain the same kind of quark ratios, it is anticipated that two ratios have similar behavior in the intermediate transverse momentum region. We expect to obtain useful information on constituent quarks in different hadrons by comparing the ratio of normal hadrons produced by recombination in heavy ion collisions, the baryon/meson ratio, to the ratio between the four-quark $X(3872)$ meson and the $\Xi_{c c}$ baryon, the meson/baryon ratio.

We show in Fig. 5 transverse momentum distribution ratios between the $X_{4}$ and the $\Xi_{c c}, c \bar{c} q \bar{q} / c c q$, between the $\Omega_{c c c}$ and the $\Omega_{s c c}, c c c / c c s$, between the $\Omega_{c c c}$ and the $\Xi_{c c}$, $c c c / c c q$, between the $X_{4}$ and the $\Omega_{c c c}, c \bar{c} q \bar{q} / c c c$, between the $\Omega_{s c c}$ and the $\Xi_{c c}, c c s / c c q$, and between the $X_{4}$ and the $\Omega_{s c c}, c \bar{c} q \bar{q} / c c s$ at both RHIC $\sqrt{s_{N N}}=200 \mathrm{GeV}$ and LHC $\sqrt{s_{N N}}=2.76 \mathrm{TeV}$. In the ratio the $\Xi_{c c}$ implies either a $\Xi_{c c}^{+}$or a $\Xi_{c c}^{++}$.

We note from Fig. 5 that some ratios have a peak in the intermediate transverse momentum region whereas some do not. The peak shown in transverse momentum distribution ratios looks similar to that in the transverse momentum distribution ratio between an anti-proton and a pion, which is attributable to a competition between two different hadron production mechanisms from two different quark distributions; one is an exponential at low transverse momenta and the other a power law mostly at high transverse momenta. We find that the peak appearing here in the transverse momentum distribution ratios between multi-charmed hadrons is related to the number and type of quark constituents participating in hadron production.

As introduced in Eq. (23), light quarks in quark-gluon plasma are assumed to be in thermal equilibrium with an exponential transverse momentum distribution. On the other hand charm quarks are not in thermal equilibrium in a system, and therefore transverse momentum distributions of charm quarks as shown in Eq. (22) contain a power law type in addition to an exponential transverse momentum distribution. For this reason contributions from charm quarks at higher transverse momenta prevail that from light quarks; the peak in some ratios appears when a power law type of charm quark distribution is involved with a purely exponential type light or strange quarks whose transverse momentum distributions.

It should be noted that although light quarks also have a power law type transverse momentum distribution at high transverse momentum, for such effects to be visible in hadron ratios, the momentum of a charm quark or a charmed hadron itself should have an even larger transverse momentum because the transverse momentum of heavy quark hadrons is mostly dominated by the momentum of the heavy quarks. Therefore, it is reasonable to consider only light quarks with an exponential transverse momentum distribution when we are interested in the transverse momentum distribution of a heavy quark hadron in the intermediate transverse momentum region.

The transverse momentum distribution ratio between the $\Omega_{c c c}$ and the $\Omega_{s c c}, c c c / c c s$ in Fig. 5 (b), and that between the $\Omega_{c c c}$ and the $\Xi_{c c}, c c c / c c q$ in Fig. 5] (c) both showing the peak in the intermediate transverse momentum region represents the ratio between a charm and a strange or a light quark in addition to two other common charm quarks. We see that those two ratios are similar in magnitude, $\sim 10^{-2}$ reflecting that the number of charm quarks in a system is smaller than that of light quarks by a factor of a few hundreds. The additional difference in magnitude by a factor 3 between two ratios is originated from both the number difference between strange and light quarks in the system, and the heavier mass of strange quarks compared to that of light quarks.

We also notice that the position of the peak is located at higher transverse momentum when more heavier quarks are involved; the peak in Fig. 5(b), $c / s$ is shifted to the higher transverse momentum compared to that in Fig. 5(c), c/q. The peak located at the higher transverse momentum for hadrons with heavier quarks supports the argument that the momentum of heavy quark hadrons is mostly carried by heavy quarks due to their heavier mass [38, 39]. 

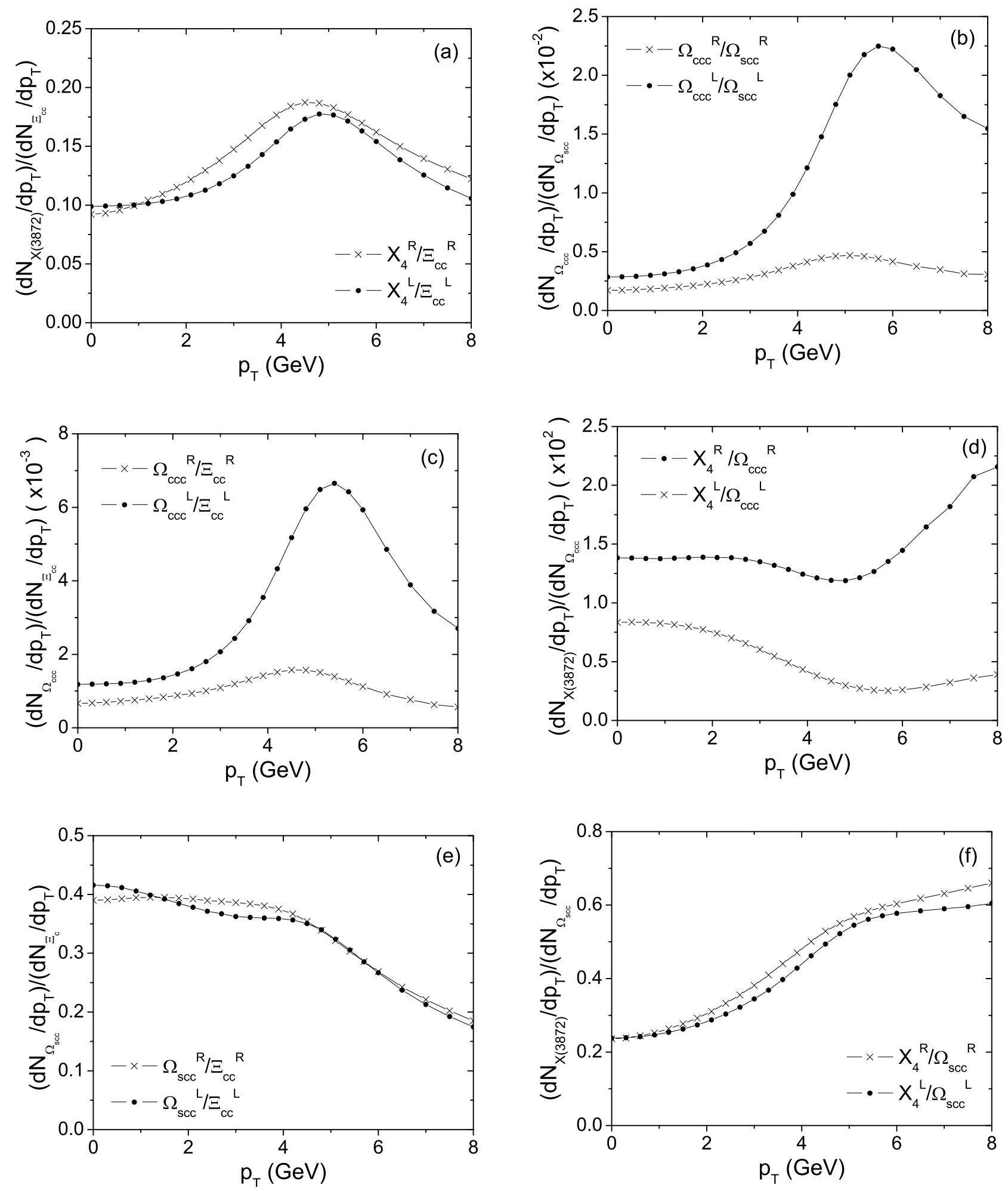

FIG. 5: Transverse momentum distribution ratios (a) between the $X(3872)$ and the $\Xi_{c c}$, (b) between the $\Omega_{c c c}$ and the $\Omega_{s c c}$, (c) between the $\Omega_{c c c}$ and the $\Xi_{c c}$, (d) between the $\Omega_{c c c}$ and the $X(3872)$, (e) between the $\Xi_{c c}$ and the $\Omega_{s c c}$, and (f) between the $X(3872)$ and the $\Omega_{s c c}$ for both RHIC $\sqrt{s_{N N}}=200 \mathrm{GeV}$ and $\mathrm{LHC} \sqrt{s_{N N}}=2.76 \mathrm{TeV}$.

In addition we further argue that the location of the peak is closely related to the transverse momentum dis- 
tribution of the spectator quark in the ratio. For example, the transverse momentum of the $\Omega_{c c c}$ is expected to be carried by three charm quarks equally on average. In case of the $\Omega_{s c c}$ with the same transverse momentum as the $\Omega_{c c c}$, the charm quark in the $\Omega_{s c c}$ will have the larger transverse momentum than that in the $\Omega_{c c c}$ on average, and therefore makes the smaller contribution to the transverse momentum distribution of the $\Omega_{s c c}$ from the higher charm quark transverse momentum, Eq. (22), or Fig. 1 compared to that in the $\Omega_{c c c}$.

Similarly, charm quarks in the $\Xi_{c c}$ with the same transverse momentum as the $\Omega_{s c c}$ has a larger transverse momentum than those in the $\Omega_{s c c}$. Therefore, the light quark in the $\Xi_{c c}$ has a smaller transverse momentum than the strange quark in the $\Omega_{s c c}$ on average, and thereby the larger contribution from the light quark transverse momentum distribution at low transverse momenta, Eq. (23), to the transverse momentum distribution of the $\Xi_{c c}$ is possible compared to the strange quark in the $\Omega_{s c c}$. On the other hand, at higher transverse momenta contribution from charm quarks becomes dominant due to the power law in transverse momentum in their transverse momentum distributions, Eq. (11). Therefore there exists both the contribution from the light and charm quark transverse momentum distribution at intermediate transverse momentum region, resulting in the peak in the transverse momentum distribution ratio between the $\Omega_{c c c}$ and the $\Xi_{c c}$.

Moreover, the charm quark in the $\Omega_{s c c}$ with the relatively smaller transverse momentum compared to that in the $\Xi_{c c}$ together with the strange quark in the $\Omega_{s c c}$ with the relatively larger transverse momentum than the light quark in the $\Xi_{c c}$ has a larger contributions also to the ratio between the $\Omega_{c c c}$ and the $\Omega_{s c c}$ at higher transverse momenta compared to the case of the $\Xi_{c c}$, and make the position of the peak a little bit shifted to the higher transverse momentum compared to the peak in the ratio between the $\Omega_{c c c}$ and the $\Xi_{c c}$.

We also see that no peaks exist in transverse momentum distribution ratios between the $\Omega_{s c c}$ and the $\Xi_{c c}$, Fig. 5 (e) and between the $X(3872)$ and the $\Omega_{s c c}$, Fig. 5 (f). The ratio in Fig. 5 (e) is about $s / q$, and that in 5 (f) is about $q \bar{q} / s$ except two spectator charm quarks. Due to the slight mass difference between light and strange quarks, $200 \mathrm{MeV}$, charm quarks in the $\Xi_{c c}$ are expected to have the transverse momentum slightly larger than that of charm quarks in the $\Omega_{s c c}$, resulting in the smaller transverse momentum for the light quark in the $\Xi_{c c}$ compared to that of the strange quark in the $\Omega_{s c c}$ for all the given transverse momenta. Therefore the contribution from the light quark in the $\Xi_{c c}$ is always larger than that from the strange quark in the $\Omega_{s c c}$, leading to the decreasing ratio in Fig. 5 (e).

The same phenomena takes place in the ratio between the $X(3872)$ and the $\Omega_{s c c}$. The transverse momentum of charm quarks in the $X(3872)$ is smaller than that of charm quarks in the $\Omega_{s c c}$ due to the mass difference between two light quarks and the strange quark, $100 \mathrm{MeV}$, making each light quark to have the smaller transverse momentum than the strange quark in the $\Omega_{s c c}$. Therefore, the contribution from two light quarks in the $X(3872)$ is always larger than that from the strange quark in the $\Omega_{s c c}$ for all the given transverse momenta, leading to the increasing ratio in Fig. 5(f).

It should be also noted that in Fig. 5(e) the ratio is expected to stop decreasing and begin to increase while the ratio in Fig. 5. (f) is expected to stop increasing and begin to decrease at very high transverse momentum region eventually. As has been mentioned above, light quarks are assumed in thermal equilibrium with only an exponential transverse momentum distribution. When light quarks actually have a power law transverse momentum distribution, the ratio between heavy hadrons containing light quarks where the remaining factors involve only light quarks like that in Fig. [5), can show peaks but at very high transverse momentum because the power law effects of light quarks are turned on at only very high transverse momentum in hadrons with heavy quarks.

The transverse momentum distribution ratio between the $X(3872)$ and the $\Omega_{c c c}$, Fig. 5 (d) leaving quarks of $q \bar{q} / c$ in the ratio also shows no peaks. We find that there can exist the peak when the inverse of the ratio, the transverse momentum distribution ratio between the $\Omega_{c c c}$ and the $X(3872), c / \bar{q} / q$ is taken. The light quark in the $X(3872)$ having the much smaller transverse momentum than charm quarks in the $\Omega_{c c c}$ has a large contribution to the transverse momentum distribution of the $X(3872)$ at low transverse momenta, resulting in the decreasing ratio in Fig. 5(d). However, at higher transverse momenta the contribution from charm quarks becomes dominant, resulting in the increasing ratio in Fig. 5 (d) with increasing transverse momenta.

In that sense the transverse momentum distribution ratio between the $X(3872)$ and the $\Xi_{c c}$, given in Fig. 5 (a), is noticeable since it has a peak in the intermediate transverse momentum region even though the ratio is again about light quarks, $q \bar{q} / q$. The transverse momentum distribution ratio between the $X(3872)$ and the $\Xi_{c c}$ is similar to the ratio between anti-protons and pions, or that between the $\Lambda_{c}$ and the $D^{0}$ since those ratios leave the same number of quarks in the ratio, $q \bar{q} / q$. The reason for the peak appearing in Fig. 5 (a) is that quarks in the ratio are quarks of the same type contrary to those in Fig. 5(f). As shown in Table IIIyield ratios between the $X(3872)$ and the $\Xi_{c c}$ are at most 0.11 at RHIC and 0.12 at LHC in the statistical hadronization model when the isospin is taken into account. We see in Fig. 5 (a) that the ratio increases up to about 0.20 at both $\mathrm{RHIC}$ and LHC, no significant enhanced production of the heavier $X(3872)$ meson compared to the $\Xi_{c c}$ baryon in the intermediate transverse momenta. 

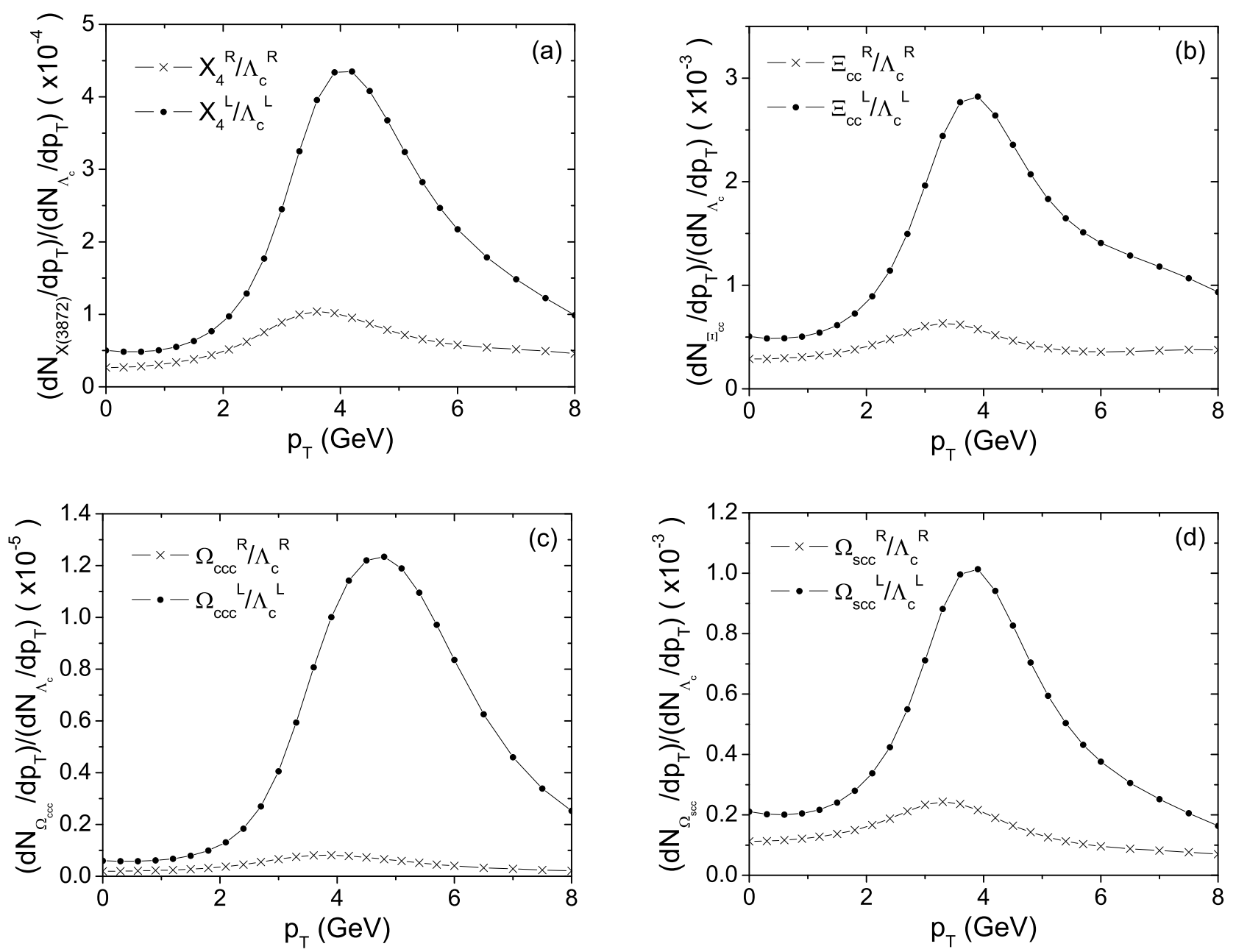

FIG. 6: Transverse momentum distribution ratios (a) between the $X(3872)$ and the $\Lambda_{c}$, (b) between the $\Xi_{c c}$ and the $\Lambda_{c}$, (c) between the $\Omega_{c c c}$ and the $\Lambda_{c}$, and (d) between the $\Omega_{s c c}$ and the $\Lambda_{c}$ at both RHIC $\sqrt{s_{N N}}=200 \mathrm{GeV}$ and LHC $\sqrt{s_{N N}}=2.76 \mathrm{TeV}$.

Moreover, as we see in Fig. 5 (a), (e) and (f), transverse momentum distribution ratios between the $X(3872)$ and the $\Xi_{c c}$, between the $\Omega_{s c c}$ and the $\Xi_{c c}$, and between the $X(3872)$ and the $\Omega_{s c c}$ at RHIC are very similar to those at LHC. Since the same function for the light quark transverse momentum distribution, Eq. (23) with the same effective temperature at both RHIC and LHC has been introduced in the analysis, ratios at RHIC and LHC obtained after cancelling two spectator charm quarks should be somehow similar to each other. In other words, the difference of the transverse momentum distribution ratios at RHIC and LHC shown in Figs. 5 (b), (c) and (d) must be originated from explicitly different transverse momentum distributions of charm quarks at RHIC and LHC, Eq. (22).

\section{B. Transverse momentum distribution ratios between a multi-charmed hadron and a $\Lambda_{c}$}

We compare the transverse momentum distribution of multi-charmed hadrons with that of the singly-charmed hadron, the $\Lambda_{c}$. For the $\Lambda_{c}$ transverse momentum distribution we have included the contribution of the $\Lambda_{c}$ production by fragmentation as well as feed-down contributions from $\Sigma_{c}(2455), \Sigma_{c}(2520), \Lambda_{c}(2595)$, and $\Lambda_{c}(2625)$ baryons. We show in Fig. 6 four transverse momentum distribution ratios, (a) between the $X(3872)$ and the $\Lambda_{c}$, (b) between the $\Xi_{c c}$ and the $\Lambda_{c}$, (c) between the $\Omega_{c c c}$ and the $\Lambda_{c}$, and (d) between the $\Omega_{s c c}$ and the $\Lambda_{c}$ for both RHIC, $\sqrt{s_{N N}}=200 \mathrm{GeV}$ and LHC, $\sqrt{s_{N N}}=2.76 \mathrm{TeV}$. 

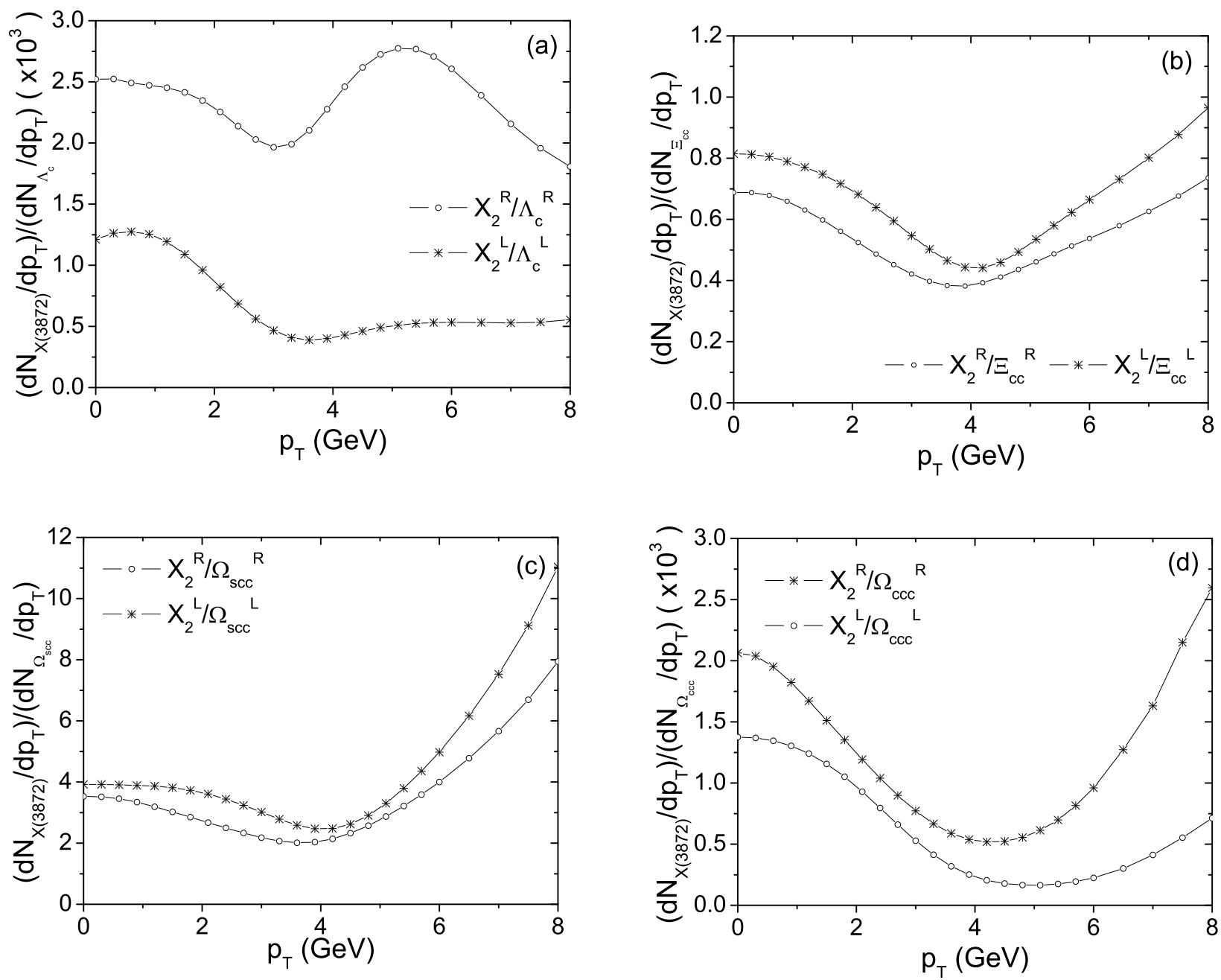

FIG. 7: Transverse momentum distribution ratios (a) between the $X(3872)$ in a two-quark state, the $X_{2}$ and the $\Lambda_{c}$, (b) between the $X_{2}$ and the $\Xi_{c c}$, (c) between the $X_{2}$ and the $\Omega_{s c c}$, and (d) between the $X_{2}$ and the $\Omega_{s c c}$ for both RHIC $\sqrt{s_{N N}}=200$ $\mathrm{GeV}$ and $\mathrm{LHC} \sqrt{s_{N N}}=2.76 \mathrm{TeV}$.

As we see in Fig. 6. the ratio is much smaller than unity, again reflecting the small possibility to coalesce more charm quarks to form a multi-charmed hadron. Since the number of charm quarks are smaller than that of light quarks by an order of two, the ratio between the $X(3872)$ and the $\Lambda_{c}$ is also smaller than that between the $X(3872)$ and the $\Xi_{c c}$ by the same order. Nevertheless we still see peaks appearing in the intermediate transverse momentum region, but at lower transverse momentum about $4 \mathrm{GeV}$.

We have argued that the peak can appear for the ratio involving both light quarks in thermal equilibrium with an exponential transverse momentum distribution and charm quarks with a power law type transverse momentum distribution in addition to an exponential transverse momentum distribution. We have also found that the peak appears in the ratio involving pure light quarks with the same kind but different numbers in the numerator and the denominator, e.g., $q \bar{q} / q$.

We further argue that a peak appears in the ratio involving charm and light quarks, especially when a remaining charm quark is in the numerator and a light quark remains in the denominator, e.g., $c / q$. As shown in Fig. 5 the peak appears in ratios, $c / s$ (b) and $c / q(\mathrm{c})$ except the peak in the ratio, $q \bar{q} / q$ (a). No peak appears for the ratio, $q / c(\mathrm{~d}), s / q(\mathrm{e})$, and $q \bar{q} / s$ (f). We see that all the ratios shown in Fig. 6 involve at least one charm quark in the numerator and one light quark in the denominator, $\bar{q} \bar{c} / q(\mathrm{a}), c / q(\mathrm{~b}), c c / q q(\mathrm{c})$, and $c s / q q$ (d), and therefore we find that the peak always appears in the ratio between a multi-charm hadron and a $\Lambda_{c}$.

Transverse momentum distribution ratios shown in Fig. 6] (a), (b) and (d) look very similar in both shape and magnitude $\sim 10^{-3}$; three ratios represents $c \bar{c} q \bar{q} / c q q$, 
$c c q / c q q$, and $c c s / c q q$, respectively. If we neglect spectator quarks we see ratios $\bar{c} \bar{q} / q, c / q$, and $c s / q q$. The inclusion of one more light quark in the numerator, $c \bar{c} q \bar{q}$ not only suppresses more the ratio $c \bar{c} q \bar{q} / c q q$ to $\sim 10^{-4}$, and also broadens the peak in Fig. [6 (a) compared to other two ratios. The same exponential transverse momentum distribution and $200 \mathrm{MeV}$ mass difference between light and strange quarks give the similar ratios, $c / q$ and $c s / q q$ as shown in Fig 6 (b) and (d).

As we see in Fig. 6 (c), the ratio between the $\Omega_{c c c}$ and the $\Lambda_{c}, c c c / c c q$ is smaller than other ratios due to one more charm quark in the numerator, the $\Omega_{c c c}$, and the peak in the $c c c / c c q$ ratio is shifted to the higher transverse momentum compared to other three ratios. If we compare all the ratios involving the $\Omega_{c c c}$, Fig. [5 (b), (c), and Fig. 6 (c), we see that the ratio with the doublycharmed hadron, the $\Xi_{c c}$ and the $\Omega_{s c c}$ has a peak at the higher transverse momentum. We also find that the ratio with the $\Omega_{c c c}$ has the broader peak than other ratios. As has been pointed out [38] this is originated from the heavy mass of charm quarks. Since the momentum of heavy quark hadrons is mostly carried out by heavy quarks, it is expected that the transverse momentum distribution would be independent of the transverse momentum at the limit of the infinite quark mass. By this reason, the ratio of the $\Omega_{c c c}$ with the doubly-charmed baryon has the broader peak at the higher transverse momentum than the ratio with the $\Lambda_{c}$.

\section{Transverse momentum distribution ratios between an $X_{2}$ and a charmed hadron}

Using the same transverse momentum distribution of charmed hadrons shown in Fig. [3] we also evaluate transverse momentum distribution ratios between the $X(3872)$ meson in a two-quark state and the charmed baryon, the $\Lambda_{c}, \Xi_{c c}, \Omega_{s c c}$, and $\Omega_{c c c}$. These are ratios between the meson and the baryon, or those between two and three quarks. The ratio between the $X_{2}$ and the $\Lambda_{c}$ is the ratio, $\bar{c} / q q$, that between the $X_{2}$ and the $\Xi_{c c}$ is the ratio, $\bar{c} / q c$, that between the $X_{2}$ and the $\Omega_{s c c}$ is the ratio, $\bar{c} / s c$, and that between the $X_{2}$ and the $\Omega_{c c c}$ is the ratio, $\bar{c} / c c$ after cancelling the spectator quarks. We show these transverse momentum distribution ratios in Fig. 7.

As shown in Fig. 7, the ratios between the $X_{2}$ and charmed baryons are completely different from those between the $X(3872)$ in a four-quark state and charmed baryons, Figs 5 and 6 . We do not see any peak in the ratio between the $X_{2}$ and the charmed baryon in Fig. 7. Moreover, the ratio shown in Fig. 7(b), (c), and (d) looks like the upside down of the ratio with the peak. We actually confirm that there exist peaks when we evaluate transverse momentum distribution ratios between the multi-charmed hadron and the $X_{2}$; the ratio between the $\Xi_{c c}$ and the $X_{2}, c q / \bar{c}$, that between the $\Omega_{s c c}$ and the $X_{2}$, $s c / \bar{c}$, and that between the $X_{2}$ and the $\Omega_{c c c}, c c / \bar{c}$. Even though we obtain the similar plot as shown in Fig. [5

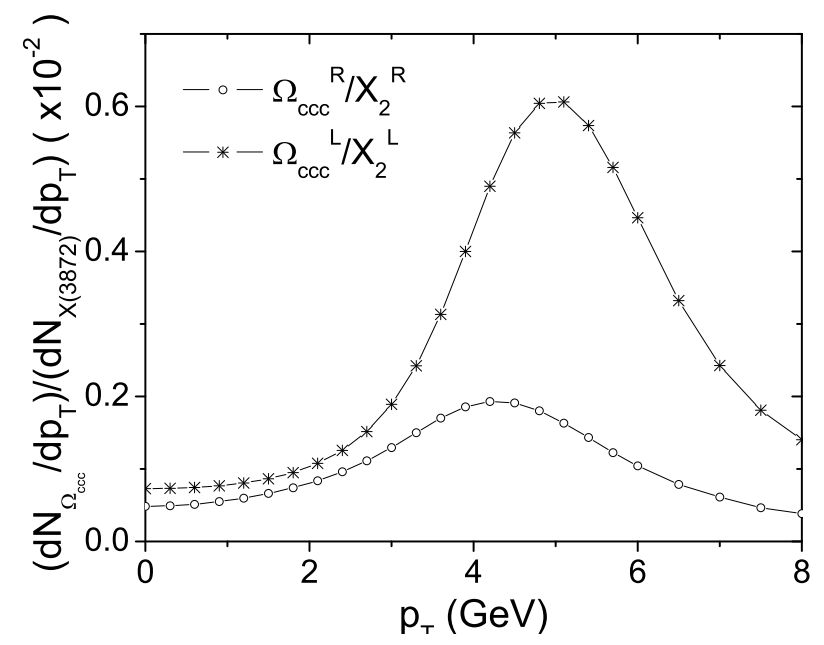

FIG. 8: The transverse momentum distribution ratio between the $\Omega_{c c c}$ and the $X(3872)$ in a two-quark state, $c c c / c \bar{c}$.

(b) if we evaluate the transverse momentum distribution ratio between the $\Omega_{s c c}$ and the $X_{2}$, we show the transverse momentum distribution ratio between the $X_{2}$ and the $\Omega_{s c c}$ as shown in Fig. 7 (c) in order to make the comparison easier, i.e., in order to consider only the ratio of the $X(3872)$ to any charmed hadrons. Nevertheless, it is interesting to observe the peak in the ratio between the $\Omega_{c c c}$ and the $X_{2}$ as shown in Fig. 8 similar to that in the ratio between the anti-proton and the pion. It must be due to similar quark contents in two ratios, $\bar{q} \bar{q} / q$ for the $\bar{p} / \pi$ and $c c / \bar{c}$ for the $\Omega_{c c c} / X_{2}$.

As expected from the transverse momentum distribution ratio between the $X(3872)$ and various charmed hadrons, the transverse momentum distribution of the $X(3872)$ meson in a two-quark state is quite different from that of the $X(3872)$ meson in a four-quark state. Therefore, we consider that we can identify whether the $X(3872)$ meson is composed of four quarks or two quarks by measuring the transverse momentum distribution ratio between the $X(3872)$ and various charmed baryons. We also show transverse momentum distributions, $d N_{X(3872)} / d p_{T}$ of both the $X(3872)$ in a four-quark sate and that in a two-quark state in Fig. 9. Recently, transverse momentum spectra of the $X(3872)$ cross section for $\mathrm{Pb}-\mathrm{Pb}$ and $\mathrm{Kr}-\mathrm{Kr}$ collisions at $\sqrt{s}=5 \mathrm{TeV}$ have been predicted in the statistical hadronization model [42]. We hope that we can compare directly the transverse momentum distribution of the $X(3872)$ yield obtained here to that measured in relativistic heavy ion collision experiments as well as that obtained in the statistical hadronization model in the near future, and that we can identify the quark structure of the $X(3872)$ meson. 


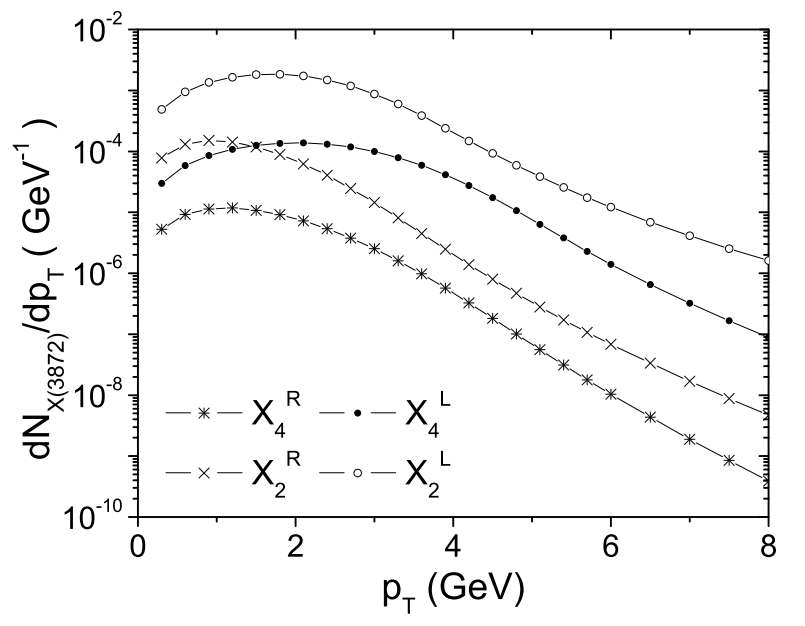

FIG. 9: Transverse momentum distributions of both the $X(3872)$ in a four-quark sate, $X_{4}$ and that in a two-quark state, $X_{2}$ for $\sqrt{s_{N N}}=200 \mathrm{GeV}$ at RHIC and $\sqrt{s_{N N}}=2.76 \mathrm{TeV}$ at LHC.

\section{CONCLUSION}

We have studied the production of multi-charmed hadrons by recombination in relativistic heavy ion collisions by focusing on the production of $\Xi_{c c}, \Xi_{c c}^{*}, \Omega_{s c c}$, $\Omega_{s c c}^{*}, \Omega_{c c c}$ baryons and $X(3872)$ mesons. We first pay attention to the yield in heavy ion collisions, and have estimated that of multi-charmed hadrons mentioned above at chemical freeze-out in both the statistical and coalescence model. We have also discussed the various yield ratio between multi-charmed hadrons.

Secondly we focus on the transverse momentum distribution in heavy ion collisions, and have evaluated that of multi-charmed hadrons, the $\Xi_{c c}, \Xi_{c c}^{*}, \Omega_{s c c}, \Omega_{s c c}^{*}, \Omega_{c c c}$ baryon and the $\mathrm{X}(3872)$ meson at mid-rapidity in the coalescence model. We have also obtained transverse momentum distribution ratios between multi-charmed hadrons, especially transverse momentum distribution ratios related to the $X(3872)$ meson, a meson/baryon ratio similar to a usual baryon/meson ratio, in order to investigate whether there exists enhanced production for a four-quark hadron compared to a normal hadron at intermediate transverse momentum region due to some reasons, e.g., hadron production mechanisms or transverse momentum distributions of constituent quarks. We have further evaluated transverse momentum distribution ratios between multi-charmed hadrons and a singly charmed baryon, the $\Lambda_{c}$. Lastly, we have discussed the transverse momentum distribution of the $X(3872)$ in a four-quark state, the $X_{4}$, and that of the $X(3872)$ in a two-quark state, the $X_{2}$.

We find that yields decrease with increasing number of charm and light quarks in multi-charmed hadrons in both the statistical and coalescence models as expected. How- ever, when the $X(3872)$ is considered to be a normal meson composed of a charm and and an anti-charm quark, the $X_{2}$, the yield in the coalescence model is almost same as that in the statistical model. It is interesting to notice that the yield of the $X_{4}$ is comparable to that of the $\Omega_{s c c}$ in the coalescence model when the feed-down contribution of the $\Omega_{s c c}^{*}$ to the $\Omega_{s c c}$ is not taken into account; the effect of including two more light quarks of the constituent mass $350 \mathrm{MeV}$ is comparable to that of adding one more strange quark of the mass $500 \mathrm{MeV}$.

The yield of multi-charmed hadrons in the quark coalescence model is found to be smaller than that in the statistical model, reflecting the suppression effects in the quark coalescence process. Among the yield ratio between charmed hadrons we find that yield ratios involving the $\Omega_{c c c}$, or $\Omega_{c c c} / \Xi_{c c}, \Omega_{c c c} / \Omega_{s c c}$ and $X_{4} / \Omega_{c c c}$ at LHC are always larger than those at RHIC in both the statistical and coalescence model. For other ratios without the $\Omega_{c c c}$ ratios at RHIC are comparable to or larger than those at LHC.

Transverse momentum distributions of charmed hadrons at LHC are found to be larger than those at RHIC due to the larger number of charm and light quarks available at LHC compared to that at RHIC. We find that the transverse momentum distribution of the $X_{4}$ meson is larger than that of the $\Omega_{c c c}$ baryon by two orders of magnitude at both RHIC and LHC. The effect from the much smaller abundance of charm quarks in the system compared to that of light quarks by a factor of hundreds at RHIC and LHC overwhelms the typical larger probability for forming a hadron composed of three charm quarks compared to the relatively smaller possibility for forming a hadron with four quarks, leading to the smaller transverse momentum distribution of the $\Omega_{c c c}$ baryon compared to that of the $X(3872)$ meson.

We note that some transverse momentum distribution ratios between multi-charmed hadrons have a peak in the intermediate transverse momentum region, similar to that in the transverse momentum distribution ratio between an anti-proton and a pion. We find that the peak appearing in the transverse momentum distribution ratio between multi-charmed baryons is related to a kind of quark constituents participating in hadron production; specific combinations between light quarks in thermal equilibrium with an exponential transverse momentum distribution and charm quarks not in thermal equilibrium in a system with their transverse momentum distributions of both a power law type and an exponential. We also notice that the position of the peak is located at higher transverse momentum when more heavier quarks are involved; the peak in the ratio $c c c / c c s$ is shifted to the higher transverse momentum compared to that in the ratio, $c c c / c c q$. The peak located at the higher transverse momentum for hadrons with heavier quarks confirms the argument that the momentum of heavy quark hadrons is mostly carried by heavy quarks due to their heavier mass. In addition we further observe that the location of the peak is closely related to the transverse momentum 
distribution of the spectator quark in the ratio.

The transverse momentum distribution ratio between the $X(3872)$ and the $\Xi_{c c}$ is noticeable since it has a peak in the intermediate transverse momentum region, leaving light quarks in the ratio, $q \bar{q} / q$ similar to the ratio between anti-protons and pions, or that between the $\Lambda_{c}$ and the $D^{0}$. We note that the ratio increases up to about 0.20 at both RHIC and LHC, no significant enhanced production of the heavier $X(3872)$ meson compared to the $\Xi_{c c}$ baryon in the intermediate transverse momentum region.

The transverse momentum distribution ratio between the multi-charmed hadron and the $\Lambda_{c}$ is found to be much smaller than unity, reflecting the small possibility to coalesce more charm quarks to form a multi-charmed hadron, but to have a peak in all the ratios. We argue that a peak appears in the ratio involving charm and light quarks, especially when a remaining charm quark is in the numerator and a light quark remains in the denominator after cancelling common quarks, e.g., $c / q$. We also note that the ratio of the $\Omega_{c c c}$ with the doubly-charmed baryon has the broader peak at the higher transverse momentum than that of the $\Omega_{c c c}$ with the $\Lambda_{c}$.

The ratio between the $X(3872)$ in a two-quark state, the $X_{2}$ and charmed baryons are calculated to be completely different from that between the $X(3872)$ in a fourquark state, the $X_{4}$ and charmed baryons. We consider that we can infer the quark content of the $X(3872)$ by measuring the transverse momentum distribution ratio between the $X(3872)$ and various charmed baryons. It is interesting to observe the peak in the ratio between the $\Omega_{c c c}$ and the $X_{2}$ similar to that in the ratio between the anti-proton and the pion, attributable to similar quark contents in two ratios, $\bar{q} \bar{q} / q$ for the $\bar{p} / \pi$ and $c c / \bar{c}$ for the $\Omega_{c c c} / X_{2}$. We hope that we compare the transverse momentum distribution of the $X(3872)$ yield obtained here to that measured in relativistic heavy ion collision experiments as well as that obtained in the statistical hadronization model in the near future, and that we identify the quark structure of the $X(3872)$ meson.

In summary we find that yields of multi-charmed hadrons in heavy ion collisions at RHIC and LHC are large enough, and thereby not only multi-charmed hadrons observed so far, e.g., the $\Xi_{c c}$ but also those which have not been observed yet, are expected to be discovered more easily in heavy ion collisions. On the other hand the transverse momentum distribution of multicharmed hadrons is found to keep the information of their constituent quarks at the moment of hadron production very well, and the effects of constituent quarks, especially charm quarks on the transverse momentum distribution of the multi-charmed hadron become more visible in the transverse momentum ratio between various multicharmed hadrons.

Charm quarks carrying most of the total momentum of charmed hadrons due to their heavier mass compared to that of light quarks, determine both the position and broadness of the peak in the transverse momentum distribution ratio between charmed quark hadrons. The transverse momentum distribution ratio reflects the interplay between quark contents of two corresponding hadrons, and the peak in the transverse momentum distribution ratio between multi-charmed hadrons appears only under certain circumstances, presenting us with meaningful information on constituent quarks. Therefore we expect that studying both transverse momentum distributions of multi-charmed hadrons themselves and transverse momentum distribution ratios between various multi-charmed hadrons provide us useful information on hadron production mechanism involving charm quarks in heavy ion collisions.

\section{Acknowledgements}

S. Cho was supported by the National Research Foundation of Korea (NRF) grant funded by the Korea government (MSIP) (No. 2016R1C1B1016270) and supported by the National Research Foundation of Korea (NRF) grant funded by the Korea government (MSIT) (No. 2018R1A5A1025563). S. H. Lee was supported by Samsung Science and Technology Foundation under Project Number SSTF-BA1901-04.

\section{Appendix A: Relative coordinates for different internal structures of the $\mathrm{X}(3872)$ meson}

When the $X(3872)$ meson is in a tetra-quark state, there exist various possibilities to define relative coordinates, and therefore relative momenta in explaining the internal structure of the $X(3872)$ meson [43]. As introduced in Sec. III, the most common space coordinates used to describe four-quark states are,

$$
\begin{aligned}
& \vec{R}=\vec{r}_{l}^{\prime}+\vec{r}_{\bar{l}}^{\prime}+\vec{r}_{c}^{\prime}+\vec{r}_{\bar{c}}^{\prime}, \\
& \vec{r}_{1}^{\prime}=\vec{r}_{l}^{\prime}-\vec{r}_{\bar{l}}^{\prime}, \\
& \vec{r}_{2}^{\prime}=\frac{m_{l} \vec{r}_{l}^{\prime}+m_{\bar{l}} \vec{r}_{\bar{l}}^{\prime}}{m_{l}+m_{\bar{l}}}-\vec{r}_{c}, \\
& \vec{r}_{3}^{\prime}=\frac{m_{l} \vec{r}_{l}^{\prime}+m_{\bar{l}} \vec{r}_{\bar{l}}^{\prime}+m_{c} \vec{r}_{c}^{\prime}}{m_{l}+m_{\bar{l}}+m_{c}}-\vec{r}_{\bar{c}}^{\prime},
\end{aligned}
$$

and the corresponding relative transverse momenta are,

$$
\begin{aligned}
& \vec{k}=\vec{p}_{l T}^{\prime}+\vec{p}_{\bar{l} T}^{\prime}+\vec{p}_{c T}^{\prime}+\vec{p}_{\bar{c} T}^{\prime}, \\
& \vec{k}_{1}=\frac{m_{\bar{l}} \vec{p}_{l T}^{\prime}-m_{l} \vec{p}_{\bar{l} T}^{\prime}}{m_{l}+m_{\bar{l}}}, \\
& \vec{k}_{2}=\frac{m_{c}\left(\vec{p}_{l T}^{\prime}+\vec{p}_{\bar{l} T}^{\prime}\right)-\left(m_{l}+m_{\bar{l}}\right) \vec{p}_{c T}^{\prime}}{m_{l}+m_{\bar{l}}+m_{c}}, \\
& \left.\vec{k}_{3}=\frac{m_{\bar{c}}\left(\vec{p}_{l T}^{\prime}+\vec{p}_{\bar{l} T}^{\prime}+\vec{p}_{c T}^{\prime}\right)-\left(m_{l}+m_{\bar{l}}+m_{c}\right) \vec{p}_{\bar{c} T}^{\prime}}{m_{l}+m_{\bar{l}}+m_{c}+m_{\bar{c}}}\right)
\end{aligned}
$$




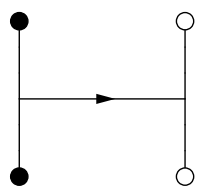

(a)

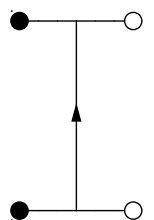

(b)

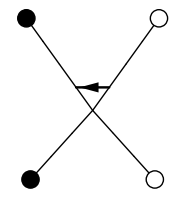

(c)
FIG. 10: Alternative relative coordinates for a four-quark $X(3872)$ meson. Filled circles represent quarks and empty circles represent anti-quarks.

with reduced masses for the above configurations,

$$
\begin{aligned}
\mu_{1} & =\frac{m_{l} m_{\bar{l}}}{m_{l}+m_{\bar{l}}}, \quad \mu_{2}=\frac{\left(m_{l}+m_{\bar{l}}\right) m_{c}}{m_{l}+m_{\bar{l}}+m_{c}} \\
\mu_{3} & =\frac{\left(m_{l}+m_{\bar{l}}+m_{c}\right) m_{\bar{c}}}{m_{l}+m_{\bar{l}}+m_{c}+m_{\bar{c}}}
\end{aligned}
$$

which describe relative coordinates of quarks in the $X(3872)$ meson; the distance between any two quarks, $\vec{r}_{1}^{\prime}$, that between the center of mass for previously chosen two quarks and the third quark, $\vec{r}_{2}^{\prime}$, and that between the center of mass for three quarks and the remaining quark fourth quark, $\vec{r}_{3}^{\prime}$. Here two light quarks are chosen for the first relative coordinate $\vec{r}_{1}^{\prime}$ in Eq. (A1) but it does not matter whether other combination of quarks, e.g., two charm quarks or one light and one charm quarks, is chosen.

On the other hand, if we want to explain the $X(3872)$ meson formed from the coalescence of two quark pairs, especially when we want to describe the most probable configuration of the $X(3872)$ meson which easily decays strongly to $J / \Psi$ and $\rho$ mesons, we find that the following relative coordinates and momenta would be more appropriate,

$$
\begin{aligned}
& \vec{R}=\vec{r}_{l}^{\prime}+\vec{r}_{\bar{l}}^{\prime}+\vec{r}_{c}^{\prime}+\vec{r}_{\bar{c}}^{\prime}, \\
& \vec{r}_{1}^{\prime}=\vec{r}_{l}^{\prime}-\vec{r}_{\bar{l}}^{\prime}, \\
& \vec{r}_{2}^{\prime}=\vec{r}_{c}^{\prime}-\vec{r}_{\bar{c}}^{\prime}, \\
& \vec{r}_{3}^{\prime}=\frac{m_{l} \vec{r}_{l}^{\prime}+m_{\bar{l}} \vec{r}_{\bar{l}}^{\prime}}{m_{l}+m_{\bar{l}}}-\frac{m_{c} \vec{r}_{c}^{\prime}+m_{\bar{c}} \vec{r}_{\bar{c}}}{m_{c}+m_{\bar{c}}},
\end{aligned}
$$

and

$$
\begin{aligned}
& \vec{k}=\vec{p}_{l T}^{\prime}+\vec{p}_{\bar{l} T}^{\prime}+\vec{p}_{c T}^{\prime}+\vec{p}_{\bar{c} T}^{\prime}, \\
& \vec{k}_{1}=\frac{m_{\bar{l}} \vec{p}_{l T}^{\prime}-m_{l} \vec{p}_{\bar{l} T}^{\prime}}{m_{l}+m_{\bar{l}}}, \\
& \vec{k}_{2}=\frac{m_{\bar{c}} \vec{p}_{c T}^{\prime}-m_{c} \vec{p}_{\bar{c} T}^{\prime}}{m_{c}+m_{\bar{c}}}, \\
& \vec{k}_{3}=\frac{\left(m_{c}+m_{\bar{c}}\right)\left(\vec{p}_{l T}^{\prime}+\vec{p}_{\bar{l} T}^{\prime}\right)-\left(m_{l}+m_{\bar{l}}\right)\left(\vec{p}_{c T}^{\prime}+\vec{p}_{\bar{c} T}^{\prime}\right)}{m_{l}+m_{\bar{l}}+m_{c}+m_{\bar{c}}},
\end{aligned}
$$

with reduced masses for the above configurations,

$$
\begin{aligned}
\mu_{1} & =\frac{m_{l} m_{\bar{l}}}{m_{l}+m_{\bar{l}}}, \quad \mu_{2}=\frac{m_{c} m_{\bar{c}}}{m_{c}+m_{\bar{c}}}, \\
\mu_{3} & =\frac{\left(m_{l}+m_{\bar{l}}\right)\left(m_{c}+m_{\bar{c}}\right)}{m_{l}+m_{\bar{l}}+m_{c}+m_{\bar{c}}} .
\end{aligned}
$$

In the coordinate, Eq. (A4), two pairs of two quarks are chosen first, and then the distance between the center of mass between two pairs of two quarks is taken. We show a diagram describing a system of relative coordinates, Eq. (A4) in Fig. 10 (b) similar to the figure as shown in Ref. [43]. Filled circles represent quarks, and empty circles represent anti-quarks, and Fig. 10 (b) and (c) correspond to meson-meson channels, a direct and an exchange channel, respectively. If the upper circles represent heavy quarks and lower circles represent the light quarks, the system of coordinates, Eq. (A4) corresponds to the direct channel good for describing the formation of the $X(3872)$ meson from the $J / \Psi$ and $\rho$ meson whereas the following exchange channel would be good for describing the decay of the $X(3872)$ meson to $D^{*}$ and $\bar{D}$ mesons or $\bar{D}^{*}$ and $D$ mesons,

$$
\begin{aligned}
& \vec{R}=\vec{r}_{l}^{\prime}+\vec{r}_{\bar{l}}^{\prime}+\vec{r}_{c}^{\prime}+\vec{r}_{\bar{c}}^{\prime}, \\
& \vec{r}_{1}^{\prime}=\vec{r}_{c}^{\prime}-\vec{r}_{\bar{l}}^{\prime}, \\
& \vec{r}_{2}^{\prime}=\vec{r}_{l}^{\prime}-\vec{r}_{\bar{c}}^{\prime}, \\
& \vec{r}_{3}^{\prime}=\frac{m_{c} \vec{r}_{c}^{\prime}+m_{\bar{l}} \vec{r}_{\bar{l}}^{\prime}}{m_{c}+m_{\bar{l}}}-\frac{m_{l} \vec{r}_{l}^{\prime}+m_{\bar{c}} \vec{r}_{\bar{c}}}{m_{l}+m_{\bar{c}}},
\end{aligned}
$$

and

$$
\begin{aligned}
& \vec{k}=\vec{p}_{l T}^{\prime}+\vec{p}_{\bar{l} T}^{\prime}+\vec{p}_{c T}^{\prime}+\vec{p}_{\bar{c} T}^{\prime}, \\
& \vec{k}_{1}=\frac{m_{\bar{l}} \vec{p}_{c T}^{\prime}-m_{c} \vec{p}_{\bar{l} T}^{\prime}}{m_{c}+m_{\bar{l}}}, \\
& \vec{k}_{2}=\frac{m_{\bar{c}} \vec{p}_{l T}^{\prime}-m_{l} \vec{p}_{\bar{c} T}^{\prime}}{m_{l}+m_{\bar{c}}}, \\
& \vec{k}_{3}=\frac{\left(m_{c}+m_{\bar{l}}\right)\left(\vec{p}_{l T}^{\prime}+\vec{p}_{\bar{c} T}^{\prime}\right)-\left(m_{l}+m_{\bar{c}}\right)\left(\vec{p}_{c T}^{\prime}+\vec{p}_{\bar{l} T}^{\prime}\right)}{m_{l}+m_{\bar{l}}+m_{c}+m_{\bar{c}}},
\end{aligned}
$$

with reduced masses for this configuration,

$$
\begin{aligned}
\mu_{1} & =\frac{m_{c} m_{\bar{l}}}{m_{c}+m_{\bar{l}}}, \quad \mu_{2}=\frac{m_{l} m_{\bar{c}}}{m_{l}+m_{\bar{c}}}, \\
\mu_{3} & =\frac{\left(m_{c}+m_{\bar{l}}\right)\left(m_{l}+m_{\bar{c}}\right)}{m_{l}+m_{\bar{l}}+m_{c}+m_{\bar{c}}} .
\end{aligned}
$$

Similarly, a system of coordinates for Fig. 10 (a) becomes,

$$
\begin{aligned}
& \vec{R}=\vec{r}_{l}^{\prime}+\vec{r}_{\bar{l}}^{\prime}+\vec{r}_{c}^{\prime}+\vec{r}_{\bar{c}}^{\prime}, \\
& \vec{r}_{1}^{\prime}=\vec{r}_{c}^{\prime}-\vec{r}_{l}^{\prime}, \\
& \vec{r}_{2}^{\prime}=\vec{r}_{\bar{l}}^{\prime}-\vec{r}_{\bar{c}}^{\prime}, \\
& \vec{r}_{3}^{\prime}=\frac{m_{c} \vec{r}_{c}^{\prime}+m_{l} \vec{r}_{l}^{\prime}}{m_{c}+m_{l}}-\frac{m_{\bar{l}} \vec{r}_{\bar{l}}^{\prime}+m_{\bar{c}} \vec{r}_{\bar{c}}^{\prime}}{m_{\bar{l}}+m_{\bar{c}}},
\end{aligned}
$$


and

$$
\begin{aligned}
& \vec{k}=\vec{p}_{l T}^{\prime}+\vec{p}_{\bar{l} T}^{\prime}+\vec{p}_{c T}^{\prime}+\vec{p}_{\bar{c} T}^{\prime}, \\
& \vec{k}_{1}=\frac{m_{l} \vec{p}_{c T}^{\prime}-m_{c} \vec{p}_{l}^{\prime}}{m_{c}+m_{l}}, \\
& \vec{k}_{2}=\frac{m_{\bar{c}} \vec{p}_{\bar{l} T}^{\prime}-m_{\bar{l}} \vec{p}_{\bar{c} T}^{\prime}}{m_{\bar{l}}+m_{\bar{c}}}, \\
& \vec{k}_{3}=\frac{\left(m_{c}+m_{l}\right)\left(\vec{p}_{\bar{l} T}^{\prime}+\vec{p}_{\bar{c} T}^{\prime}\right)-\left(m_{\bar{l}}+m_{\bar{c}}\right)\left(\vec{p}_{c T}^{\prime}+\vec{p}_{l T}^{\prime}\right)}{m_{l}+m_{\bar{l}}+m_{c}+m_{\bar{c}}},
\end{aligned}
$$

with reduced masses,

$$
\begin{aligned}
\mu_{1} & =\frac{m_{c} m_{l}}{m_{c}+m_{l}}, \quad \mu_{2}=\frac{m_{\bar{l}} m_{\bar{c}}}{m_{\bar{l}}+m_{\bar{c}}}, \\
\mu_{3} & =\frac{\left(m_{c}+m_{l}\right)\left(m_{\bar{l}}+m_{\bar{c}}\right)}{m_{l}+m_{\bar{l}}+m_{c}+m_{\bar{c}}} .
\end{aligned}
$$

As has been discussed in Refs. 17 19 it has been found that the yield of an exotic hadron depends on the internal structure of the exotic hadron. We therefore consider that a transverse momentum distribution of an exotic hadron should be somehow dependent on its internal structure. Moreover, we have alternative relative coordinates in describing a four-quark hadron, and therefore we need to check whether the transverse momentum distribution of an exotic hadron, here the $X(3872)$ meson would be dependent also on each relative coordinate.

When we look at the equation for the transverse momentum distribution of the $X(3872)$ meson, Eq. (14), we see that the only part where the relative coordinates play an important role is the Wigner function. As has been pointed out in Ref. [30] different wave functions contribute to the transverse momentum distribution differently through the Wigner function for hadrons composed of the same kind and number of constituents. If we just consider here the Gaussian Wigner function in a $s$-wave applied in the analysis, Eq. (14), we find that the relative momentum part becomes,

$$
e^{-\sigma_{1}^{2} k_{1}^{2}-\sigma_{2}^{2} k_{2}^{2}-\sigma_{3}^{2} k_{3}^{2}}=e^{-\frac{1}{\omega_{c}}\left(\frac{k_{1}^{2}}{\mu_{1}}+\frac{k_{2}^{2}}{\mu_{2}}+\frac{k_{3}^{2}}{\mu_{3}}\right)} .
$$

with the relation, $\sigma^{2}=1 / \mu \omega$. When we put relative momenta corresponding to alternative relative coordinates, Eqs. (A1), (A4), (A7), and (A10) with the reduced mass also corresponding to each relative coordinate, we obtain,

$$
-\sigma_{1}^{2} k_{1}^{2}-\sigma_{2}^{2} k_{2}^{2}-\sigma_{3}^{2} k_{3}^{2}=-\frac{1}{\omega_{c}}\left(\frac{k_{1}^{2}}{\mu_{1}}+\frac{k_{2}^{2}}{\mu_{2}}+\frac{k_{3}^{2}}{\mu_{3}}\right)
$$

$$
\begin{aligned}
& =-\frac{1}{\omega_{c}} \frac{1}{m_{l}+m_{\bar{l}}+m_{c}+m_{\bar{c}}}\left(\frac{m_{\bar{l}}+m_{c}+m_{\bar{c}}}{m_{l}} p_{l T}^{\prime}{ }^{2}\right. \\
& +\frac{m_{c}+m_{\bar{c}}+m_{l}}{m_{\bar{l}}} p_{\bar{l} T}^{\prime}{ }^{2}+\frac{m_{\bar{c}}+m_{l}+m_{\bar{l}}}{m_{c}} p_{c T}^{\prime}{ }^{2} \\
& +\frac{m_{l}+m_{\bar{l}}+m_{c}}{m_{\bar{c}}} p_{\bar{c} T}^{\prime}{ }^{2}-2\left(\vec{p}_{l T}^{\prime} \cdot \vec{p}_{\bar{l} T}^{\prime}+\vec{p}_{\bar{l} T}^{\prime} \cdot \vec{p}_{c T}^{\prime}\right. \\
& \left.\left.+\vec{p}_{c T}^{\prime} \cdot \vec{p}_{\bar{c} T}^{\prime}+\vec{p}_{l T}^{\prime} \cdot \vec{p}_{c T}^{\prime}+\vec{p}_{\bar{l} T}^{\prime} \cdot \vec{p}_{c T}^{\prime}+\vec{p}_{\bar{l} T}^{\prime} \cdot \vec{p}_{\bar{c} T}^{\prime}\right)\right)(\mathrm{A} 14)
\end{aligned}
$$

the same result regardless of any relative coordinates; the argument in Eq. (A14) is symmetric between four quarks, and thus does not change under any exchange between constituent quarks in the $X(3872)$ meson. Therefore, we find that the transverse momentum distribution for a four-quark state does not depend on the choice of relative coordinates. In other words, we cannot identify the internal structure of a hadron as shown in Fig. 10 based on transverse momentum distributions of the hadron. We, however, still see that if the four-quark state has the internal relative momentum, e.g., $p$-wave or $d$-wave, and so on, then the transverse momentum distribution would be dependent on it since there exists an additional term outside the argument in the exponential Wigner function as shown in Eq. (20), and thereby the yield is expected to be affected by the relative internal momentum as discussed in Refs. [17 -19].

We also show the argument similar to that shown in Eq. (A14) for three-quark baryons,

$$
\begin{aligned}
& -\sigma_{1}^{2} k_{1}^{2}-\sigma_{2}^{2} k_{2}^{2}=-\frac{1}{\omega}\left(\frac{k_{1}^{2}}{\mu_{1}}+\frac{k_{2}^{2}}{\mu_{2}}\right) \\
& =-\frac{1}{\omega} \frac{1}{m_{1}+m_{2}+m_{3}}\left(\frac{m_{1}+m_{2}}{m_{3}} p_{3 T}^{\prime}{ }^{2}\right. \\
& +\frac{m_{2}+m_{3}}{m_{1}} p_{1 T}^{\prime}{ }^{2}+\frac{m_{3}+m_{1}}{m_{2}} p_{2 T}^{\prime 2} \\
& \left.-2\left(\vec{p}_{1 T}^{\prime} \cdot \vec{p}_{2 T}^{\prime}+\vec{p}_{2 T}^{\prime} \cdot \vec{p}_{3 T}^{\prime}+\vec{p}_{3 T}^{\prime} \cdot \vec{p}_{1 T}^{\prime}\right)\right),
\end{aligned}
$$

which is symmetric between three quarks, and thus does not change under any exchange between constituent quarks in the three-quark baryon. We also see that if the three-quark baryon has the internal relative momentum, then the transverse momentum distribution would be dependent on it, and thereby the yield is expected to be affected by the relative internal momentum.
[1] J. Adams et al. (STAR Collaboration), Nucl. Phys. A 757, 102 (2005).

[2] K. Adcox et al. (PHENIX Collaboration), ibid., 184 (2005).
[3] M. Gyulassy and L. McLerran, Nucl. Phys. A 750, 30 (2005).

[4] S. Gupta, X. Luo, B. Mohanty, H. G. Ritter and N. Xu, Science 332, 1525 (2011). 
[5] T. Matsui and H. Satz, Phys. Lett. B 178, 416 (1986).

[6] H. Satz, J. Phys. G 32, R25 (2006).

[7] F. Karsch, D. Kharzeev and H. Satz, Phys. Lett. B 637, 75 (2006).

[8] A. Mocsy and P. Petreczky, Phys. Rev. Lett. 99, 211602 (2007).

[9] P. Braun-Munzinger and J. Stachel, Phys. Lett. B 490, 196 (2000).

[10] R. L. Thews, M. Schroedter and J. Rafelski, Phys. Rev. C 63, 054905 (2001).

[11] A. Andronic, P. Braun-Munzinger, K. Redlich and J. Stachel, Phys. Lett. B 652, 259 (2007).

[12] V. Greco, C. M. Ko, and P. Levai, Phys. Rev. Lett. 90, 202302 (2003).

[13] V. Greco, C. M. Ko, and P. Levai, Phys. Rev. C 68, 034904 (2003).

[14] R. J. Fries, B. Muller, C. Nonaka, and S. A. Bass, Phys. Rev. Lett. 90, 202303 (2003).

[15] R. J. Fries, B. Muller, C. Nonaka, and S. A. Bass, Phys. Rev. C 68, 044902 (2003).

[16] D. Molnar and S. A. Voloshin, Phys. Rev. Lett. 91, 092301 (2003).

[17] S. Cho et al. (ExHIC Collaboration), Phys. Rev. Lett. 106, 212001 (2011).

[18] S. Cho et al. (ExHIC Collaboration), Phys. Rev. C 84, 064910 (2011).

[19] S. Cho et al. (ExHIC Collaboration), Prog. Part. Nucl. Phys. 95, 279 (2017).

[20] A. De Rujula, H. Georgi and S. L. Glashow, Phys. Rev. D 12, 147 (1975).

[21] M. Mattson et al. (SELEX Collaboration), Phys. Rev. Lett. 89, 112001 (2002).

[22] R. Aaij et al. (LHCb Collaboration), Phys. Rev. Lett. 119, no. 11, 112001 (2017).

[23] J. Zhao, H. He and P. Zhuang, Phys. Lett. B 771, 349 (2017).

[24] X. Yao and B. Muller, Phys. Rev. D 97, no. 7, 074003 (2018).
[25] S. K. Choi et al. (Belle Collaboration), Phys. Rev. Lett. 91, 262001 (2003).

[26] M. Nielsen, F. S. Navarra and S. H. Lee, Phys. Rept. 497, 41 (2010).

[27] S. Cho and S. H. Lee, Phys. Rev. C 88, 054901 (2013).

[28] A. Martinez Torres, K. P. Khemchandani, F. S. Navarra, M. Nielsen and L. M. Abreu, Phys. Rev. D 90, no. 11, 114023 (2014).

[29] M. Cleven, V. K. Magas and A. Ramos, arXiv:1906.06116 [hep-ph].

[30] S. Cho, Phys. Rev. C 91, no. 5, 054914 (2015).

[31] A. Andronic, P. Braun-Munzinger, J. Stachel, Nucl. Phys. A 772, 167 (2006).

[32] A. Andronic, P. Braun-Munzinger, K. Redlich and J. Stachel, Nucl. Phys. A 904-905, 535c (2013).

[33] J. Stachel, A. Andronic, P. Braun-Munzinger and K. Redlich, J. Phys. Conf. Ser. 509, 012019 (2014).

[34] R. A. Briceno, H. W. Lin and D. R. Bolton, Phys. Rev. D 86, 094504 (2012).

[35] J. Beringer et al. (Particle Data Group Collaboration), Phys. Rev. D 86, 010001 (2012)

[36] M. Karliner and J. L. Rosner, Phys. Rev. D 97, no. 9, 094006 (2018).

[37] R. Scheibl and U. W. Heinz, Phys. Rev. C 59, 1585 (1999).

[38] Y. Oh, C. M. Ko, S. H. Lee, and S. Yasui, Phys. Rev. C 79, 044905 (2009).

[39] S. Plumari, V. Minissale, S. K. Das, G. Coci and V. Greco, Eur. Phys. J. C 78, no. 4, 348 (2018).

[40] J. Adam et al. [STAR Collaboration], Phys. Rev. C 99, no. 3, 034908 (2019).

[41] J. Adam et al. [ALICE Collaboration], JHEP 1603, 081 (2016).

[42] A. Andronic, P. Braun-Munzinger, M. K. Kohler, K. Redlich and J. Stachel, arXiv:1901.09200 [nucl-th].

[43] D. M. Brink and F. Stancu, Phys. Rev. D 49, 4665 (1994). 